\title{
Pyridinium p-DSSC Dyes: An Old Acceptor Learns New Tricks
}

\author{
Anil Reddy Marri, ${ }^{\mathrm{a}}$ Fiona A. Black, ${ }^{\mathrm{b}}$ John Mallows, ${ }^{\mathrm{b}}$ Elizabeth A. Gibson ${ }^{\mathrm{b}}$ and John Fielden ${ }^{\mathrm{a} *}$ \\ ${ }^{a}$ School of Chemistry, University of East Anglia, Norwich Research Park, Norwich, NR4 7TJ, \\ United Kingdom.
}

${ }^{\mathrm{b}}$ School of Chemistry, Newcastle University, Newcastle-upon-Tyne, NE1 7RU, UK.

\begin{abstract}
A family of six (five new) thiophenyl bridged triarylamine-donor based dyes with pyridine anchoring groups have been synthesized and studied as sensitizers for the p-type dye-sensitized solar cell (p-DSSC). They comprise bis-dicyano acceptor systems with a single pyridyl binder incorporated directly into the triarylamine (1), or separated by a phenyl group (2); a monodicyano with two phenyl pyridine binders (3); and respective homologues 4 to $\mathbf{6}$ with pyridinium acceptors. In all cases, compared to their dicyano counterparts, the pyridinium based dyes have higher extinction coefficients and smaller HOMO-LUMO gaps that give broader spectrum absorption. Thus, despite lower dye uptake, devices based on pyridiniums 4 and $\mathbf{6}$ have identical power conversion efficiencies $(\eta)$ to the equivalent dicyano systems $\mathbf{1}$ and 3. However, the best performing device $(\eta=0.06 \%)$ is based on the known bis-acceptor dicyano system $\mathbf{2}$, as the large size and double positive charge of $\mathbf{5}$ leads to a substantial disadvantage in loading on $\mathrm{NiO}$. Absorbed-photon-to-current efficiencies for $\mathbf{5}$ are competitive with or higher than those of $\mathbf{2}$, implying a better per dye performance consistent with the absorption profile, and DFT calculations suggesting better charge separation. Thus, pyridiniums may provide a new, and easily accessible high performance acceptor for p-DSSC dyes, but are likely better paired with anionic binding groups such as carboxylates.
\end{abstract}




\section{Introduction}

Dye Sensitized solar cells have attracted great attention since the first report of Grätzel and coworkers in 1991,[1] as low cost alternatives to conventional silicon solar cells.[2,3] To date, the best efficiency obtained is around $13 \%$, reported by Grätzel et. al, based on panchromatic dyes with cobalt electrolytes in n-type DSSCs.[4] In the n-DSSC architecture, the ability of dyes to usefully harvest low energy photons is limited by the need to combine a HOMO low enough in energy to be regenerated by the redox mediator, and a LUMO high enough to efficiently inject electrons into the conduction band.[5] A potential solution is to build tandem DSSCs, where a photoactive cathode would replace the platinised counter electrode of the Grätzel cell and harvest lower energy photons, increasing both spectral coverage and photovoltage $\left(V_{\mathrm{OC}}\right) \cdot[5-8]$

Over the past decade, extensive research has been conducted into the p-type dye sensitized solar cells (p-DSSCs) needed to pair with n-DSSCs in tandem devices.[5-15] In a p-type DSSC, the operating mechanism is the reverse of that of the n-type DSSC (i.e, Grätzel solar cells): instead of injecting electrons into an n-type semiconductor upon photoexcitation, the sensitizer dye injects holes to the valence band (VB) of a p-type semiconductor (typically $\mathrm{NiO}$ ). Then the redox electrolyte oxidizes the reduced dye molecule and transfers electrons to the counter electrode (or n-type DSSC photoanode). However, the power conversion efficiencies (PCEs) of p-DSSCs have remained very low, preventing construction of useful tandem devices. This is due to a number of factors, including the small energy difference between the VB of $\mathrm{NiO}$ and the redox mediator, rapid back transfer of photogenerated holes from $\mathrm{NiO}$ to the electrolyte, and slow hole transfer through NiO.[5-8] Changing redox electrolyte from $\mathrm{I}^{-} / \mathrm{I}_{3}{ }^{-}$to $\mathrm{Co}^{2+/ 3+}[9,10]$ and latterly $\mathrm{Fe}^{2+/ 3+}[11]$ based systems has led to greatly increased $V_{\mathrm{OC}}$ (up to 709 $\mathrm{mV}$ ) and the highest p-DSSC power conversion efficiencies (PCE, $\eta$ ) to date (up to $2.5 \%$ ), but the negative redox potentials associated with these mediators would severely reduce the 
photovoltage produced by the $\mathrm{TiO}_{2}$ side of any tandem cell. With the more $\mathrm{TiO}_{2}$ compatible $\mathrm{I}^{-}$ $/ \mathrm{I}_{3}{ }^{-}$, the p-DSSC record $V_{\mathrm{OC}}$ remains $350 \mathrm{mV}$,[12] and record $\eta 0.61 \%$.[13], and there are only a few reports of pn-tandem systems whose overall performance exceeds that of the n-type component alone.[14]

Dye-sensitized solar cells are complex devices whose overall performance can be unpredictable, so development relies on a combination of design, and exploration of new materials. To date, nearly all sensitizers for p-DSSCs have used the carboxylic acid anchoring groups employed in n-DSSCs, but one report has suggested pyridyl binders could be competitive, and give high Voc.[15] Pyridyl groups may also be more suitable for binding alternative p-SCs. Similarly, while dicyano acceptor groups have featured in many p-DSSC dyes, cationic acceptor groups - such as the indoliums that have achieved record $J_{\mathrm{SC}}[16]$ - have been little explored. One large, and easily accessible family of cationic organic acceptor groups are the pyridiniums, whose absorption properties can easily be tailored by adjusting the quaternising group - for example by replacing alkyl groups with phenyl, or dinitrophenyl. [17] Herein, we investigate a family of six pyridyl functionalized triaryl amine donor-acceptor dyes with both dicyano and methyl pyridinium acceptor groups, with either two anchors and one acceptor or one anchor and two acceptors, and pyridine groups either directly forming part of the triaryl amine or appended to one of the phenyl groups (Fig. 1). While the pyridiniums give higher extinction coefficients and absorb across a broader spectrum of light, and DFT calculations suggest better spatial separation of HOMO and LUMO (and thus charge) the best device performance is obtained from the bis-dicyano, mono-pyridyl architecture. This appears to be a consequence of poor uptake of the larger, and cationic pyridinium dyes at the positively charged $\mathrm{NiO}$ surface, thus suggesting that charge compensation (for example from carboxylate binders) could yield a new class of easily accessible and high performance p-DSSC dyes. 

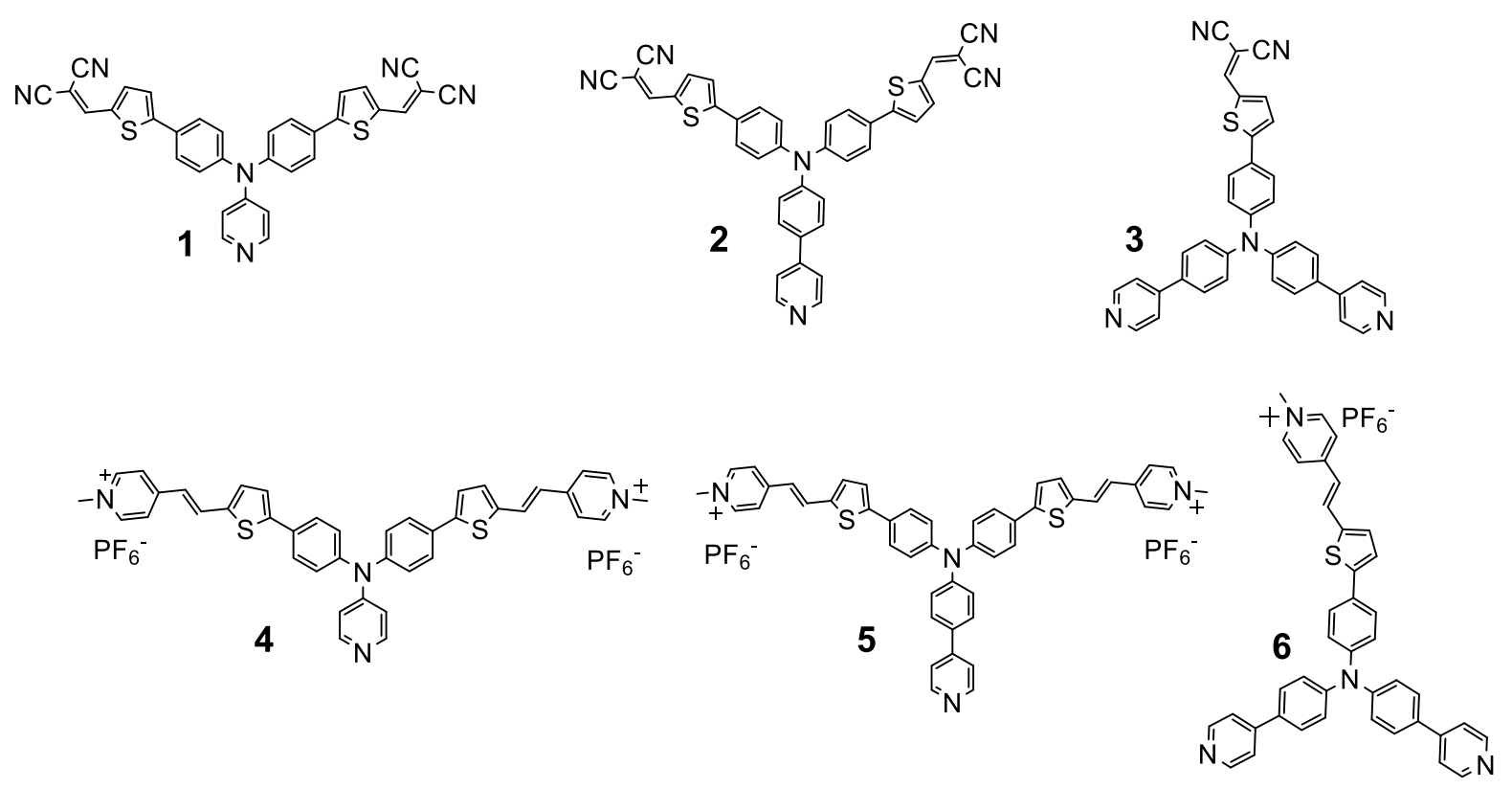

Fig. 1. Chemical structures of the dyes $\mathbf{1}$ to $\mathbf{6}$ used in this study. Compound $\mathbf{2}$ was previously published. ${ }^{14}$

\section{Experimental}

Materials and Procedures. The starting materials 4-bromotriphenylamine, pyridine-4boronic acid dihydrate, tetrakis(triphenylphosphine) palladium(0), bis(diphenylphosphinoferrocene) palladium(II) chloride, formylthiophene boronic acid, Nbromosuccinimide, 4-methyl pyridine, iodomethane and malononitrile were purchased from Sigma-Aldrich and Alfa Aesar. Dry dimethylformamide (DMF) for electrochemical experiments was purchased from Sigma-Aldrich in SureSeal ${ }^{\circledR}$ bottles. Solvents were purified by standard procedures and purged with argon or nitrogen before use. All other chemicals used in this work were of analytical grade and were used without further purification until or unless stated, and all reactions were performed under an inert atmosphere of argon or nitrogen. $\mathrm{N}$ methyl-4-picolinium iodide,[18] 5-[4-(diphenylamino)phenyl]thiophene-2-carbaldehyde (P7)[19], 5-[4-bis-(4-bromophenyl)aminophenyl]thiophene-2-carbaldehyde (P8)[20] and the electrolyte $\left.\mathrm{N}\left(\mathrm{C}_{4} \mathrm{H}_{9}-n\right)_{4}\right] \mathrm{BF}_{4}$ [21] were synthesized according to literature procedures. Chromatographic separations were carried out on silica gel (60-120 mesh). 
General Physical Measurements. ${ }^{1} \mathrm{H}$ NMR and ${ }^{13} \mathrm{C}$ NMR spectra were recorded a Bruker Ascend $500 \mathrm{MHz}$ spectrometer. Elemental analyses and mass spectrometry were outsourced to London Metropolitan University, and the UK National Mass Spectrometry Service at Swansea University respectively. Electronic absorption spectra were recorded on an Agilent Cary60 UV-Vis spectrophotometer.. Electrochemical data were obtained using Autolab PGSTAT30 and PGSTAT302 potentiostat/galvanostats. A single-compartment cell was used with an Ag wire pseudo reference electrode, platinum working electrode and Pt wire auxiliary electrode, the supporting electrolyte was $\left[\mathrm{N}\left(\mathrm{C}_{4} \mathrm{H}_{9}-n\right)_{4}\right] \mathrm{BF}_{4}$ in dry DMF. Solutions containing ca. $10^{-3} \mathrm{M}$ analyte $\left(0.1 \mathrm{M}\right.$ electrolyte) were degassed by purging with nitrogen. All $E_{1 / 2}$ values were calculated from $\left(E_{\mathrm{pa}}+E_{\mathrm{pc}}\right) / 2$ at a scan rate of $100 \mathrm{mV} \mathrm{s} \mathrm{s}^{-1}$ and referenced to $\mathrm{Fc} / \mathrm{Fc}^{+}$.

Solar Cell Assembly and Measurements. A NiO precursor solution was prepared by dissolving anhydrous $\mathrm{NiCl}_{2}(1 \mathrm{~g})$ and the tri-block co-polymer F108 (poly(ethylene glycol)block-poly (propylene glycol)-block-poly(ethylene glycol)) (1 g) in ethanol (6 g) and distilled water $(5 \mathrm{~g})$. The solution was allowed to rest for 10 days and then centrifuged to separate out any remaining solids.

For the photocathodes, conducting glass substrates (Pilkington TEC15, sheet resistance 15 $\Omega /$ square) were cut to size (approx. $2 \times 2 \mathrm{~cm}$ ) and then cleaned by sonic bath for 15 minutes, first in soapy water, second in $0.1 \mathrm{M} \mathrm{HCl}$, and finally in ethanol. The $\mathrm{NiO}$ electrodes were made by applying the precursor solution onto the conducting glass substrates by doctor blade using Scotch Magic tape as a spacer $\left(0.2 \mathrm{~cm}^{2}\right.$ active area), followed by sintering in a Nabertherm B150 oven at $450{ }^{\circ} \mathrm{C}$ for $30 \mathrm{~min}$. Additional layers of precursor solution were applied and sintered to increase the film thickness. The $\mathrm{NiO}$ electrodes were soaked in a dichloromethane (or acetonitrile) solution of the dye $(0.3 \mathrm{mM})$ for $16 \mathrm{~h}$ at room temperature. For the counter electrodes, the conducting glass substrates (Pilkington TEC8, sheet resistance $8 \Omega /$ square $)$ were cut and cleaned as described above. A small $(\mathrm{ca} .1 \times 1 \mathrm{~mm})$ hole was drilled 
into a corner for the electrolyte solution to be introduced at the assembly stage. Platinized counter electrodes were prepared by applying chloroplatinic acid solution $(4.4 \mathrm{mM}, 10 \mu \mathrm{L} \mathrm{cm}-$ ${ }^{2}$ ) in ethanol onto the conducting glass substrate. The electrodes were then heated in a Nabertherm B150 oven at $400{ }^{\circ} \mathrm{C}$ for 15 minutes and then allowed to cool. The dyed electrodes were removed from the dye bath and washed with acetonitrile. Cells were assembled face-toface with the platinized counter electrode using a $30 \mu \mathrm{m}$ thick thermoplastic frame (Surlyn 1702). The electrolyte contained LiI $(1.0 \mathrm{M})$ and $\mathrm{I}_{2}(0.1 \mathrm{M})$ in dry acetonitrile, and was introduced through the predrilled hole in the counter electrode by vacuum back filling. The cells were then sealed using a $30 \mu \mathrm{m}$ thick thermoplastic square (Surlyn 1702) and a glass cover slip.

Current density vs. voltage measurements were measured using an Ivium CompactStat potentiostat either in the dark or under AM 1.5 simulated sunlight from an Oriel $150 \mathrm{~W}$ solar simulator, calibrated using a Si diode to give light with an intensity of $100 \mathrm{~mW} \mathrm{~cm}{ }^{-2}$. DSCs were held in place using a custom made cell holder with a circular aperture with a diameter 1 mm wider than the working area of the cell. An applied voltage was swept from slightly above the given open-circuit voltage, at a scan rate of $3 \mathrm{mV} \mathrm{s}^{-1}$. Incident photon-to- current conversion efficiencies (IPCEs) were recorded by passing the light from the solar simulator through an Oriel Cornerstone $1301 / 8 \mathrm{~m}$ monochromator over the range of $400-700 \mathrm{~nm}$ at $10 \mathrm{~nm}$ increments and recording the current from the solar cell with an Ivium CompactStat potentiostat which was calibrated against a Si photodiode.

Dye loading measurements were performed by soaking four $\mathrm{NiO}$ films for each dye in a ca. $0.3 \mathrm{mM}$ solutions of the dye in acetonitrile. UV-vis absorption spectra were taken of the films before and after soaking. To minimise the effect of any variable scattering, both bare and dyed $\mathrm{NiO}$ films were normalised to 0 abs at $800 \mathrm{~nm}$, and the spectrum of the bare film subtracted from the dyed film. The average loading was then obtained using the dye extinction coefficient. 


\section{DFT calculations}

DFT calculations were carried out using the ADF suite of programs.[22] Both geometry optimsation and property calculations were carried out using the B3LYP functional (20\% Hartree-Fock exchange)[23] and the ADF double- $\zeta$, single polarisation function DZP basis set.

This is comparable to the commonly used $6-31 \mathrm{~g}^{*}$ basis set, and initial optimization of the calculations on compounds $\mathbf{1}$ and $\mathbf{4}$ indicated that the larger triple- $\zeta$ and augmented basis sets (TZP, AUG-DZP) increased computational expense, without signficantly changing results. A 'small' frozen core was employed for the geometry optimizations. Calculations of the electronic spectra were carried out by TD-DFT using the EXCITATION modules implemented in the ADF program [24] and were based on the optimized geometries, with no frozen core. The EXCITEDGO module was used to calculate geometries of the first excited states, and ground-to-excited state dipole moment changes $\left(\Delta \mu_{12}\right)$ calculated by subtracting the ground state dipole moment from the excited state dipole moment. Solvent (acetonitrile) was introduced using COSMO with Klamt atomic Radii. The subkey NOCSMRSP was applied to prevent induced electronic charges (which respond on a much slower timescale than electronic excitations) from influencing COSMO surface charges, together with the ALLPOINTS key.

\section{Syntheses}

\section{N,N-diphenylpyridin-4-amine (P1)}

Diphenylamine (0.500 g, $2.954 \mathrm{mmol})$, 4-bromopyridine hydrochloride (0.690 g, $3.545 \mathrm{mmol})$, and $\mathrm{Pd}(\mathrm{OAc})_{2}(0.045 \mathrm{~g}, 0.12 \mathrm{mmol}),(t-\mathrm{Bu})_{3} \mathrm{P}(0.04 \mathrm{~g}, 0.12 \mathrm{mmol})$, and $t-\mathrm{BuONa}(0.852 \mathrm{~g}, 8.86$ mmol) were dissolved in toluene $(40 \mathrm{~mL})$ and stirred for $10 \mathrm{~h}$ at $100{ }^{\circ} \mathrm{C}$. After concentrating under reduced pressure, the resulting residue was dissolved in dichloromethane and washed with water. The residue was chromatographed on silica gel (hexane/ethylacetate $=3: 7$ as 
eluent) to give $\mathbf{P 1}$ as colourless solid with $34 \%$ yield. ${ }^{1} \mathrm{H} \mathrm{NMR}\left(\mathrm{CDCl}_{3}, 500 \mathrm{MHz}, \delta\right): 8.23$ (d, $J=6.6 \mathrm{~Hz}, 2 \mathrm{H}), 7.35(\mathrm{t}, J=7.8 \mathrm{~Hz}, 4 \mathrm{H}), 7.17-7.20(\mathrm{~m}, 6 \mathrm{H}), 6.73(\mathrm{~d}, J=6.6 \mathrm{~Hz}, 2 \mathrm{H}) .{ }^{13} \mathrm{C} \mathrm{NMR}$ $\left(\mathrm{CDCl}_{3}, 125 \mathrm{MHz}, \delta\right): 153.70,150.18,145.25,129.79,126.69,125.55,112.78$. ESI-MS: $m / z$ : 247.12. Elemental analysis for $\mathrm{C}_{17} \mathrm{H}_{14} \mathrm{~N}_{2}$, Anal. (Calcd) \%: C 82.78 (82.90); H 5.60 (5.73); $\mathrm{N}$ $11.25(11.37)$.

\section{N,N-bis(4-bromophenyl)pyridin-4-amine (P2)}

Compound P1 (0.300 g, $1.218 \mathrm{mmol})$ and N-bromosuccinimide (0.477 g, $2.68 \mathrm{mmol})$ were added to chloroform $(30 \mathrm{~mL})$ and several drops of acetic acid were then added. The mixture was stirred at room temperature for an hour and refluxed overnight, before cooling to room temperature and addition of water $(50 \mathrm{~mL})$. The mixture was extracted with $\mathrm{CH}_{2} \mathrm{Cl}_{2}(3 \times 50$ $\mathrm{mL}$ ) and the combined organic layers dried over $\mathrm{MgSO}_{4}$. The solvent was removed under reduced pressure and the residue was purified by column chromatography with hexane/ethylacetate (3:7) as the eluent. The product $\mathbf{P 2}$ was obtained as a colourless solid with 75\% yield. ${ }^{1} \mathrm{H} \mathrm{NMR}\left(\mathrm{CDCl}_{3}, 500 \mathrm{MHz}, \delta\right): 8.27(\mathrm{~d}, J=6.8 \mathrm{~Hz}, 2 \mathrm{H}), 7.51(\mathrm{~d}, J=8.8 \mathrm{~Hz}, 4 \mathrm{H})$, $7.05(\mathrm{~d}, J=8.8 \mathrm{~Hz}, 4 \mathrm{H}), 6.77(\mathrm{~d}, J=6.8 \mathrm{~Hz}, 2 \mathrm{H}) .{ }^{13} \mathrm{C} \mathrm{NMR}\left(\mathrm{CDCl}_{3}, 125 \mathrm{MHz}, \delta\right): 153.01$, 150.54, 144.08, 133.05, 127.88, 118.75, 113.53. ESI-MS (m/z): 404.94. Elemental analysis for $\mathrm{C}_{17} \mathrm{H}_{12} \mathrm{~N}_{2} \mathrm{Br}_{2} \bullet 0.1 \mathrm{C}_{6} \mathrm{H}_{14}$, Anal. (Calcd) \%: C, 51.10 (51.22); H 3.31 (3.27); N 6.61 (6.78).

\section{N,N-bis(5-formylthiophenyl)pyridin-4-amine (P3)}

A $50 \mathrm{~mL}$ Schlenk tube was charged with 5-formylthiophene boronic acid $(0.085 \mathrm{~g}, 0.544$ mmol), $\mathrm{Pd}\left(\mathrm{PPh}_{3}\right)_{4}(0.006 \mathrm{~g}, 0.005 \mathrm{mmol})$, dimethoxyethane $(6 \mathrm{~mL})$ and $2 \mathrm{M}$ aqueous sodium carbonate $(1.5 \mathrm{~mL})$, before purging with argon gas for 5 evacuate/refill cycles. Then compound $\mathbf{P 2}(0.100 \mathrm{~g}, 0.247 \mathrm{mmol})$ was added. The tube was sealed and heated at $90{ }^{\circ} \mathrm{C}$ with very vigorous stirring for $16 \mathrm{~h}$. Upon cooling to ambient temperature, the organics were removed by extracting three times with DCM. The combined organic fractions were washed 
with brine and dried over $\mathrm{MgSO}_{4}$. The solvent was removed under reduced pressure and the residue was purified by silica gel column chromatography using hexane/ethylacetate $(3 / 7, \mathrm{v} / \mathrm{v})$ as eluent to give $\mathbf{P 3}$ as a yellow powder in $52 \%$ yield. ${ }^{1} \mathrm{H} \mathrm{NMR}\left(300 \mathrm{MHz}, \mathrm{CDCl}_{3}, \delta\right.$ ): 9.89 (s, 2H), $8.36(\mathrm{~d}, J=7.0 \mathrm{~Hz}, 2 \mathrm{H}), 7.75(\mathrm{~d}, J=4.0 \mathrm{~Hz}, 2 \mathrm{H}), 7.67(\mathrm{~d}, J=9.0 \mathrm{~Hz}, 4 \mathrm{H}), 7.39$ (d, $J=$ $4.0 \mathrm{~Hz}, 2 \mathrm{H}), 7.24(\mathrm{~d}, J=9.0 \mathrm{~Hz}, 4 \mathrm{H}), 6.91(\mathrm{~d}, J=7.0 \mathrm{~Hz}, 2 \mathrm{H}) .{ }^{13} \mathrm{C} \mathrm{NMR}\left(75 \mathrm{MHz}, \mathrm{CDCl}_{3}, \delta\right)$ : $182.71,153.09,152.90,150.60,146.03,142.53,137.47,130.13,127.92,126.43,124.78$, 124.08, 114.78. ESI-MS (m/z): 467.0872. Elemental analysis for $\mathrm{C}_{27} \mathrm{H}_{18} \mathrm{~N}_{2} \mathrm{O}_{2} \mathrm{~S}_{2}$, Anal. (Calcd) \%: C (69.62) 69.51; H 3.95 (3.89); N 5.91 (6.00).

\section{N,N-diphenyl-4-(pyridin-4-yl)aniline (P4)}

A $50 \mathrm{~mL}$ Schlenk tube was charged with 4-pyridyl boronic acid (0.568 g, $4.626 \mathrm{mmol})$, $\mathrm{Pd}\left(\mathrm{PPh}_{3}\right)_{4}(0.178 \mathrm{mg}, \quad 0.154 \mathrm{mmol})$, dimethoxyethane $(10 \mathrm{~mL})$ and $2 \mathrm{M}$ aqueous sodium carbonate $(2.5 \mathrm{~mL})$, and was purged with argon gas for 5 evacuate/refill cycles. Then 4bromotriphenylamine $(0.500 \mathrm{~g}, 1.542 \mathrm{mmol})$ was added. The tube was sealed and heated at $90{ }^{\circ} \mathrm{C}$ with very vigorous stirring for $16 \mathrm{~h}$. Upon cooling to ambient temperature, the mixture was extracted three times with DCM. The combined organic fractions were washed with brine and dried over $\mathrm{MgSO}_{4}$. The solvent was removed under reduced pressure and the residue was purified by silica gel column chromatography using hexane/ethylacetate $(7 / 3$, v/v) as eluent to give $\mathbf{P 4}(77 \%)$ as a pale yellow powder. ${ }^{1} \mathrm{H} \mathrm{NMR}\left(500 \mathrm{MHz}, \mathrm{CDCl}_{3}, \delta\right): 8.61(\mathrm{~d}, J=6.2 \mathrm{~Hz}$, 2H), $7.52(\mathrm{~d}, J=8.8 \mathrm{~Hz}, 2 \mathrm{H}), 7.47(\mathrm{~d}, J=6.2 \mathrm{~Hz}, 2 \mathrm{H}), 7.29(\mathrm{~m}, 4 \mathrm{H}), 7.16-7.12(\mathrm{~m}, 6 \mathrm{H}), 7.08$ $(\mathrm{tt}, J=7.4 \mathrm{~Hz}, 2 \mathrm{H}) .{ }^{13} \mathrm{C} \mathrm{NMR}\left(125 \mathrm{MHz}, \mathrm{CDCl}_{3}, \delta\right): 150.14,148.99,147.71,147.26,130.92$, $129.45,127.66,125.01,123.63,122.91,120.89$. ESI-MS (m/z): 323.1542. Elemental analys is for $\mathrm{C}_{23} \mathrm{H}_{18} \mathrm{~N}_{2}$, Anal. (Calcd) \%: C 85.61 (85.68); H 5.58 (5.63); N 8.78 (8.69).

\section{4-iodo-N-(4-iodophenyl)-N-(4-(py ridin-4-yl)phenyl)aniline (P5)}


A $1 \mathrm{M}$ solution of iodine monochloride in DCM $(6.20 \mathrm{~mL}, 6.20 \mathrm{mmol})$ was added to $5 \mathrm{~mL}$ of dioxane. Zinc acetate was added to the above solution, which was stirred for 15 minutes at room temperature. Compound $\mathbf{P 4}(0.500 \mathrm{~g}, 1.551 \mathrm{mmol})$ was dissolved in $5 \mathrm{~mL}$ of dioxane and added slowly, and stirring was continued at room temperature overnight. After the reaction completed (monitored by TLC), the reaction mixture was poured into sodium thiosulfate solution (1 M, $30 \mathrm{~mL})$ and extracted twice with dichloromethane. The combined organic fractions were dried over $\mathrm{MgSO}_{4}$, then filtered and the volume reduced in vacuo. The crude product was further purified by silica gel column chromatography using hexane/ethylacetate $(7 / 3, \mathrm{v} / \mathrm{v})$ as eluent to give compound P5 $(91 \%)$ as a pale yellow powder. ${ }^{1} \mathrm{H}$ NMR $(500 \mathrm{MHz}$, $\left.\mathrm{CDCl}_{3}, \delta\right): 8.63(\mathrm{~d}, J=6.2 \mathrm{~Hz}, 2 \mathrm{H}), 7.57(\mathrm{~d}, J=8.8 \mathrm{~Hz}, 4 \mathrm{H}), 7.53(\mathrm{~d}, J=8.8 \mathrm{~Hz}, 2 \mathrm{H}), 7.47(\mathrm{~d}$, $J=6.2 \mathrm{~Hz}, 2 \mathrm{H}), 7.13(\mathrm{~d}, J=8.7 \mathrm{~Hz}, 2 \mathrm{H}), 6.87(\mathrm{~d}, J=8.8 \mathrm{~Hz}, 4 \mathrm{H}) .{ }^{13} \mathrm{C} \mathrm{NMR}\left(125 \mathrm{MHz}, \mathrm{CDCl}_{3}\right.$, $\delta): 150.06,147.81,147.57,146.62,138.54,132.41,128.02,126.44,123.95,121.04,86.88$. ESI-MS (m/z): 574.95. Elemental analysis for $\mathrm{C}_{23} \mathrm{H}_{16} \mathrm{~N}_{2} \mathrm{I}_{2}$, Anal. (Calcd) \%: C 48.19 (48.11); H 2.81 (2.81); N 4.94 (4.88).

\section{4-\{Bis-[4-(5-formyl-thiophen-2-yl)-phenyl]-amino\}-N,N-diphenyl-4-(py ridin-4-yl)aniline} (P6)

A $50 \mathrm{~mL}$ Schlenk tube was charged with 5 -formylthiophene boronic acid $(0.060 \mathrm{~g}, 0.383$ mmol), $\mathrm{Pd}\left(\mathrm{PPh}_{3}\right)_{4}(0.040 \mathrm{mg}, 0.035 \mathrm{mmol})$, dimethoxyethane $(8 \mathrm{~mL})$ and $2 \mathrm{M}$ aqueous sodium carbonate $(2.0 \mathrm{~mL})$, and purged with argon gas for 5 evacuate/refill cycles. Then compound P5 $(0.100 \mathrm{~g}, 0.174 \mathrm{mmol})$ was added. The tube was sealed and heated at $90{ }^{\circ} \mathrm{C}$ with very vigorous stirring for $16 \mathrm{~h}$. Upon cooling to ambient temperature, the mixture was extracted three times with DCM. The combined organic fractions were washed with brine and dried over $\mathrm{MgSO}_{4}$. The solvent was removed under reduced pressure and the residue was purified by silica gel column chromatography using hexane/ethylacetate $(3 / 7, \mathrm{v} / \mathrm{v})$ as eluent to give compound 
P6 (56\%) as a yellow powder. ${ }^{1} \mathrm{H}$ NMR $\left(500 \mathrm{MHz}, \mathrm{CDCl}_{3}, \delta\right): 9.88$ (s, 2H), 8.61-8.70 (br s, 2H), $7.73(\mathrm{~d}, J=3.9 \mathrm{~Hz}, 2 \mathrm{H}), 7.61(\mathrm{~m}, 6 \mathrm{H}), 7.52(\mathrm{~d}, J=6.2 \mathrm{~Hz}, 2 \mathrm{H}), 7.35(\mathrm{~d}, J=3.9 \mathrm{~Hz}, 2 \mathrm{H})$, $7.26(\mathrm{~d}, J=8.7 \mathrm{~Hz}, 2 \mathrm{H}), 7.19(\mathrm{~d}, J=8.7 \mathrm{~Hz}, 4 \mathrm{H}) .{ }^{13} \mathrm{C} \mathrm{NMR}(125 \mathrm{MHz}, \mathrm{CDCB}, \delta): 182.68$, $153.73,149.91,147.76,147.68,147.55,142.00,137.62,133.17,128.28,128.23,127.65$, 125.00, 124.53, 123.53, 121.19. ESI-MS (m/z): 543.12. Elemental analysis for $\mathrm{C}_{33} \mathrm{H}_{22} \mathrm{~N}_{2} \mathrm{O}_{2} \mathrm{~S}_{2} \bullet 1.2 \mathrm{H}_{2} \mathrm{O}$, Anal. (Calcd) \%: C 70.18 (70.24); H 4.16 (4.36); N 5.03 (4.96).

\section{5-\{4-[bis(4-pyridin-4-yl)phenyl]aminophenyl\}-thiophene-2-carbaldehyde (P9)}

A $50 \mathrm{~mL}$ of Schlenk tube was charged with 4-pyridyl boronic acid (0.096 g, $0.78 \mathrm{mmol})$, $\mathrm{Pd}\left(\mathrm{PPh}_{3}\right)_{4}(0.045 \mathrm{~g}, \quad 0.039 \mathrm{mmol})$, dimethoxyethane $(8 \mathrm{~mL})$ and $2 \mathrm{M}$ aqueous sodium carbonate $(2.0 \mathrm{~mL})$, then the tube was purged with argon gas for 5 evacuate/refill cycles. Compound P8 (0.100 g, $0.195 \mathrm{mmol})$ was and the tube was sealed and heated at $90{ }^{\circ} \mathrm{C}$ with very vigorous stirring for $16 \mathrm{~h}$. Upon cooling to ambient temperature, the mixture was extracted three times with DCM. The combined organic fractions were washed with brine and dried over $\mathrm{MgSO}_{4}$. The solvent was removed under reduced pressure and the residue was purified by silica gel column chromatography using hexane/ethylacetate $(2 / 8, v / v)$ as eluent to give compound P9 $(65 \%)$ as a yellow powder. ${ }^{1} \mathrm{H} \mathrm{NMR}\left(500 \mathrm{MHz}, \mathrm{CDCl}_{3}, \delta\right): 9.88$ (s, 1H), 8.66 (br s, 4H), $7.73(\mathrm{~d}, J=4.1 \mathrm{~Hz}, 1 \mathrm{H}), 7.59-7.62(\mathrm{~m}, 6 \mathrm{H}), 7.50(\mathrm{~d}, J=5.2 \mathrm{~Hz}, 4 \mathrm{H}), 7.35(\mathrm{~d}, J=4.0 \mathrm{~Hz}, 1 \mathrm{H})$, $7.26(\mathrm{~d}, J=8.7 \mathrm{~Hz}, 4 \mathrm{H}), 7.20(\mathrm{~d}, J=8.8 \mathrm{~Hz}, 2 \mathrm{H}) .{ }^{13} \mathrm{C} \mathrm{NMR}\left(125 \mathrm{MHz}, \mathrm{CDCl}_{3}, \delta\right): 182.66$, $153.84,150.33,148.04,147.64,147.32,141.93,137.62,133.19,128.14,127.98,127.59$, 124.90, 124.28, 123.44, 121.12. ESI-MS (m/z): 510.16. Elemental analysis for $\mathrm{C}_{33} \mathrm{H}_{23} \mathrm{~N}_{3} \mathrm{OS}$, Anal. (Calcd) \%: C 77.65 (77.78); H 4.61 (4.55); N 8.13 (8.25). 
A mixture of compound $\mathbf{P 3}(0.050 \mathrm{~g}, 0.107 \mathrm{mmol})$, malononitrile $(0.142 \mathrm{~g}, 2.14 \mathrm{mmol})$ and pyridine $(0.2 \mathrm{~mL})$ in anhydrous toluene $(6 \mathrm{~mL})$ was deoxygenated five times by placing under vacuum and backfilling with argon, and then heated at $70{ }^{\circ} \mathrm{C}$ for $16 \mathrm{~h}$. After cooling to room temperature, the solvent was removed and the residue was purified by column chromatography over silica with a dichloromethane/methanol mixture (9:1) as the eluent to give $\mathbf{1}$ as a black solid (0.035 g, 60\%). ${ }^{1} \mathrm{H} \mathrm{NMR}\left(500 \mathrm{MHz}, \mathrm{CDCl}_{3}, \delta\right) 8.40(\mathrm{~d}, J=6.3 \mathrm{~Hz}, 2 \mathrm{H}), 7.80(\mathrm{~s}, 2 \mathrm{H}), 7.7$ $(\mathrm{d}, J=4.2 \mathrm{~Hz}, 2 \mathrm{H}), 7.7(\mathrm{~d}, J=8.7 \mathrm{~Hz}, 4 \mathrm{H}), 7.42(\mathrm{~d}, J=4.1 \mathrm{~Hz}, 2 \mathrm{H}), 7.24(\mathrm{~d}, J=8.7 \mathrm{~Hz}, 4 \mathrm{H})$, $6.93(\mathrm{~d}, J=6.3 \mathrm{~Hz}, 2 \mathrm{H}) .{ }^{13} \mathrm{C} \mathrm{NMR}\left(125 \mathrm{MHz}, \mathrm{CDCl}_{3}, \delta\right): 155.15,152.62,150.87,150.44$, $146.68,140.09,134.31,129.15,128.19,126.26,124.54,115.46,114.11,113.33$. ESI-MS (m/z): 563.11. Elemental analysis for $\mathrm{C}_{33} \mathrm{H}_{18} \mathrm{~N}_{6} \mathrm{~S}_{2}$, Anal. (Calcd) \%: C 70.29 (70.44); H 3.31 (3.22); N 15.09 (14.94).

\section{4-(Bis-\{4-[5-(2,2-dicyano-vinyl)-thiophene-2-yl]-phenyl\}-amino)-4-phenylpy ridine (2)}

A mixture of compound $\mathbf{P 6}(0.060 \mathrm{~g}, 0.110 \mathrm{mmol})$, malononitrile $(0.146 \mathrm{~g}, 2.21 \mathrm{mmol})$ and pyridine $(0.2 \mathrm{~mL})$ in anhydrous toluene $(6 \mathrm{~mL})$ was deoxygenated five times by placing under vacuum and backfilling with argon, and then heated at $70{ }^{\circ} \mathrm{C}$ for $16 \mathrm{~h}$. After cooling to room temperature, the solvent was removed and the residue was purified by column chromatography over silica gel by using dichloromethane/methanol mixture $(9: 1)$ as the eluent to give $\mathbf{2}(75 \%)$ as a red solid. ${ }^{1} \mathrm{H}$ NMR $\left(500 \mathrm{MHz},\left(\mathrm{CD}_{3}\right)_{2} \mathrm{CO}, \delta\right): 8.63(\mathrm{~d}, J=6.2 \mathrm{~Hz}, 2 \mathrm{H}), 8.41(\mathrm{~s}, 2 \mathrm{H}), 7.97$ $(\mathrm{d}, J=4.2 \mathrm{~Hz}, 2 \mathrm{H}), 7.88(\mathrm{~m}, 6 \mathrm{H}), 7.72(\mathrm{~d}, J=4.2 \mathrm{~Hz}, 2 \mathrm{H}), 7.68(\mathrm{~d}, J=6.2 \mathrm{~Hz}, 2 \mathrm{H}), 7.35(\mathrm{~d}, J$ $=8.7 \mathrm{~Hz}, 2 \mathrm{H}), 7.28(\mathrm{~d}, J=8.8 \mathrm{~Hz}, 4 \mathrm{H}) .{ }^{13} \mathrm{C} \mathrm{NMR}\left(125 \mathrm{MHz},\left(\mathrm{CD}_{3}\right)_{2} \mathrm{CO}, \delta: 155.22,151.65\right.$, $150.43,148.40,147.21,146.63,141.82,134.05,133.79,128.29,127.94,127.43,125.68$, $124.59,124.42,120.80,114.38,113.74,75.25$. ESI-MS $(\mathrm{m} / \mathrm{z}): 639.14$. Elemental analysis for $\mathrm{C}_{39} \mathrm{H}_{22} \mathrm{~N}_{6} \mathrm{~S}_{2} \bullet 0.6 \mathrm{H}_{2} \mathrm{O}$,Anal. (Calcd): C 72.07 (72.11); H 3.74 (3.60); N 12.54 (12.94). 


\section{5-\{4-[bis(4-pyridin-4-yl)phenyl]aminophenyl\}-thiophene-2-vinyldinitrile (3)}

A mixture of compound $\mathbf{P 9}(0.100 \mathrm{~g}, 0.196 \mathrm{mmol})$, malononitrile $(0.130 \mathrm{~g}, 1.962 \mathrm{mmol})$ and pyridine $(0.2 \mathrm{~mL})$ in anhydrous toluene $(6 \mathrm{~mL})$ was deoxygenated five times by placing under vacuum and backfilling with argon, and then heated at $70{ }^{\circ} \mathrm{C}$ for $16 \mathrm{~h}$. After cooling to room temperature, the solvent was removed and the residue was purified by column chromatography over silica with a dichloromethane/methanol mixture (9:1) as the eluent to give compound 3 (75\%) a brown solid. ${ }^{1} \mathrm{H}$ NMR (500 MHz, $\left.\mathrm{CDCl}_{3}, \delta\right): 8.66$ (d, $\left.J=5.8 \mathrm{~Hz}, 4 \mathrm{H}\right), 7.77(\mathrm{~s}, 1 \mathrm{H})$, $7.70(\mathrm{~d}, J=4.2 \mathrm{~Hz}, 1 \mathrm{H}), 7.61-7.63(\mathrm{~m}, 6 \mathrm{H}), 7.50(\mathrm{~d}, J=6.2 \mathrm{~Hz}, 4 \mathrm{H}), 7.38(\mathrm{~d}, J=4.2 \mathrm{~Hz}, 1 \mathrm{H})$, $7.27(\mathrm{~d}, J=8.7 \mathrm{~Hz}, 4 \mathrm{H}), 7.19(\mathrm{~d}, J=8.8 \mathrm{~Hz}, 2 \mathrm{H}) .{ }^{13} \mathrm{C} \mathrm{NMR}\left(125 \mathrm{MHz}, \mathrm{CDCl}_{3}, \delta\right): 156.23$, $150.35,148.82,147.37,147.29,140.29,133.66,133.61,128.23,127.86,126.71,125.23$, 123.82, 123.72, 121.12, 114.35, 113.54. ESI-MS (m/z): 558.1735. Elemental analysis for $\mathrm{C}_{36} \mathrm{H}_{23} \mathrm{~N}_{5} \mathrm{~S} \cdot \mathrm{H}_{2} \mathrm{O}$, Anal. (Calcd) \%: C 75.16 (75.10); H 4.35 (4.38); N 12.18 (12.17).

\section{4-(Bis-\{4-[5-(2-[N-methyl-4-py ridiniumyl-vinyl)-thiophene-2-yl]-phenyl\}-amino)-}

\section{pyridine dihexafluorophosphate (4)}

A $50 \mathrm{~mL}$ round bottom flask was charged with compound $\mathbf{P 3}(0.050 \mathrm{~g}, 0.107 \mathrm{mmol})$ and $\mathrm{N}$ methylpicolinium iodide $(0.063 \mathrm{~g}, 0.268 \mathrm{mmol})$ and a 1:1 mixture of chloroform and ethanol was added $(10 \mathrm{~mL})$. A catalytic amount of piperidine was added and the mixture was refluxed at $80{ }^{\circ} \mathrm{C}$ for $4 \mathrm{hrs}$, before cooling to room temperature and addition of an excess of solid $\mathrm{NH}_{4} \mathrm{PF}$. The mixture was stirred until the product precipitated. Compound $4(0.025 \mathrm{~g}, 25 \%$, pale brown) was collected by vacuum filtration and washed several times with water and then diethyl ether. ${ }^{1} \mathrm{H}$ NMR $\left(500 \mathrm{MHz}, \mathrm{CD}_{3} \mathrm{CN}, \delta\right): 8.41(\mathrm{~d}, J=7.0 \mathrm{~Hz}, 4 \mathrm{H}), 8.30(\mathrm{dd}, J=4.8 \mathrm{~Hz}$, 2H), $7.96(\mathrm{~d}, J=16.1 \mathrm{~Hz}, 1 \mathrm{H}), 7.93(\mathrm{~d}, J=7.1 \mathrm{~Hz}, 2 \mathrm{H}), 7.74(\mathrm{~d}, J=8.6 \mathrm{~Hz}, 4 \mathrm{H}), 7.47(\mathrm{~m}, 2 \mathrm{H})$, $7.27(\mathrm{~d}, J=8.7 \mathrm{~Hz}, 4 \mathrm{H}), 7.07(\mathrm{~d}, J=16.0 \mathrm{~Hz}, 2 \mathrm{H}), 6.89(\mathrm{dd}, J=4.7 \mathrm{~Hz}, 2 \mathrm{H}), 4.17(\mathrm{~s}, 3 \mathrm{H}) .{ }^{13} \mathrm{C}$ NMR (125 MHz, $\left(\mathrm{CD}_{3} \mathrm{CN}, \delta\right):$ 153.82, 149.80, 148.68, 148.59, 148.40, 147.86, 145.05 139.83, 
$134.70,134.46,132.76,129.19,128.88,127.69,125.39,125.18,125.09,123.84,121.69$, 121.53, 47.67. ESI-MS (m/z): $323.11\left[\mathrm{M}-2 \mathrm{PF}_{6}\right]^{2+}$. Elemental analysis for $\mathrm{C}_{41} \mathrm{H}_{34} \mathrm{~F}_{12} \mathrm{~N}_{4} \mathrm{P}_{2} \mathrm{~S}_{2}$, Anal. (Calcd) \%: C 52.57 (52.49); H 3.66 (4.49); N 5.98 (5.37).

\section{4-(Bis-\{4-[5-(2-[N-methyl-4-py ridiniumyl-vinyl)-thiophene-2-yl]-phenyl\}-amino)-4- phenylpyridine dihexafluorophosphate (5)}

A $50 \mathrm{~mL}$ round bottom flask was charged with compound $\mathbf{P 6}(0.060 \mathrm{mg}, 0.110 \mathrm{mmol})$ and Nmethyl picolinium iodide $(0.057 \mathrm{~g}, 0.1841 \mathrm{mmol})$, which were then dissolved in 50:50 chloroform:ethanol $(10 \mathrm{~mL})$. A catalytic amount of piperidine was added and the mixture was refluxed at $80{ }^{\circ} \mathrm{C}$ for $4 \mathrm{~h}$. After cooling to room temperature, excess solid $\mathrm{NH}_{4} \mathrm{PF}_{6}$ was added and the mixture was stirred until precipitation occurred. The precipitate was collected by vacuum filtration and washed several times with water and diethyl ether to yield $\mathbf{5}(45 \%)$ as a dark red solid. ${ }^{1} \mathrm{H}$ NMR $\left(500 \mathrm{MHz}, \mathrm{CD}_{3} \mathrm{CN}, \delta\right): 8.61(\mathrm{~d}, J=6.2 \mathrm{~Hz}, 2 \mathrm{H}), 8.39(\mathrm{~d}, J=6.9 \mathrm{~Hz}$, 4H), $7.96(\mathrm{~d}, J=16.1 \mathrm{~Hz}, 2 \mathrm{H}), 7.92(\mathrm{~d}, J=6.9 \mathrm{~Hz}, 4 \mathrm{H}), 7.74(\mathrm{~d}, J=8.8 \mathrm{~Hz}, 2 \mathrm{H}), 7.69(\mathrm{~d}, J=$ $8.8 \mathrm{~Hz}, 4 \mathrm{H}), 7.61(\mathrm{~d}, J=6.2 \mathrm{~Hz}, 2 \mathrm{H}), 7.44(\mathrm{~m}, 4 \mathrm{H}), 7.25(\mathrm{~d}, J=8.9 \mathrm{~Hz}, 2 \mathrm{H}), 7.18(\mathrm{~d}, J=8.7$ $\mathrm{Hz}, 4 \mathrm{H}), 7.05(\mathrm{~d}, J=16.0 \mathrm{~Hz}, 2 \mathrm{H}), 4.16(\mathrm{~s}, 6 \mathrm{H}) .{ }^{13} \mathrm{C} \mathrm{NMR}\left(125 \mathrm{MHz}, \mathrm{CD}_{3} \mathrm{CN}, \delta\right): 150.45$, $148.43,145.05,134.71,134.47,128.77,127.67,125.30,125.20,125.05,123.84,121.53,47.67$. $\operatorname{ESI-MS}(m / z): 361.13\left[\mathrm{M}-2 \mathrm{PF}_{6}\right]^{2+}$. Elemental analysis for $\mathrm{C}_{47} \mathrm{H}_{38} \mathrm{~N}_{5} \mathrm{~F}_{12} \mathrm{~N}_{4} \mathrm{P}_{2} \mathrm{~S}_{2}$, Anal. (Calcd) \%: C 55.73 (55.80); H 3.78 (3.92); N 5.53 (5.45).

\section{5-\{4-[bis(4-pyridin-4-yl)phenyl]aminophenyl\}-thiophene-2-N-methylpy ridinium hexafluorophosphate (6)}

A $50 \mathrm{~mL}$ round bottom flask was charged with compound $\mathbf{P 9}(0.050 \mathrm{~g}, 0.098 \mathrm{mmol})$ and $\mathrm{N}$ methyl picolinium iodide $(0.057 \mathrm{~g}, 0.245 \mathrm{mmol})$ and 1:1 chloroform:ethanol (10 $\mathrm{mL})$ was added. A catalytic amount of piperidine was added to the above mixture, which was refluxed 
at $80{ }^{\circ} \mathrm{C}$ for $4 \mathrm{~h}$. The mixture was cooled to room temperature and excess solidNH $\mathrm{H}_{4} \mathrm{PF}_{6}$ was added, before stirring until precipitation occurred. The precipitate was collected by vacuum filtration and washed several times with water and ether to yield 6 as a red solid $(52 \%) .{ }^{1} \mathrm{H}$ NMR (500 MHz, CD 3 CN, $\delta): 8.60(\mathrm{~d}, J=6.1 \mathrm{~Hz}, 4 \mathrm{H}), 8.37(\mathrm{~d}, J=6.8 \mathrm{~Hz}, 2 \mathrm{H}), 7.93(\mathrm{~d}, J=$ $16.1 \mathrm{~Hz}, 1 \mathrm{H}), 7.91(\mathrm{~d}, J=7.0 \mathrm{~Hz}, 2 \mathrm{H}), 7.75(\mathrm{~d}, J=8.7 \mathrm{~Hz}, 4 \mathrm{H}), 7.69-7.66(\mathrm{~m}, 6 \mathrm{H}), 7.44(\mathrm{~m}$, 2H), $7.24(\mathrm{~d}, J=8.7 \mathrm{~Hz}, 4 \mathrm{H}), 7.19(\mathrm{~d}, J=8.7 \mathrm{~Hz}, 2 \mathrm{H}), 7.04(\mathrm{~d}, J=15.9 \mathrm{~Hz}, 2 \mathrm{H}), 4.16(\mathrm{~s}, 3 \mathrm{H})$.

${ }^{13} \mathrm{C}$ NMR (125 MHz, (CD $\left.3 \mathrm{CN}, \delta\right): 153.82,149.80,148.68,148.59,148.40,147.86,145.05$ $139.83,134.70,134.46,132.76,129.19,128.88,127.69,125.39,125.18,125.09,123.84$, 121.69, 121.53, 47.67. ESI-MS $(\mathrm{m} / \mathrm{z}): 599.23\left[\mathrm{M}-\mathrm{PF}_{6}\right]^{+}$. Elemental analysis for $\mathrm{C}_{40} \mathrm{H}_{31} \mathrm{~F}_{6} \mathrm{~N}_{4} \mathrm{PS} \bullet \mathrm{H}_{2} \mathrm{O}$, Anal. (Calcd) \%: C 62.89 (62.99); H 4.32 (4.36); N 7.41 (7.34).

\section{Results and discussion}

\section{Synthesis}

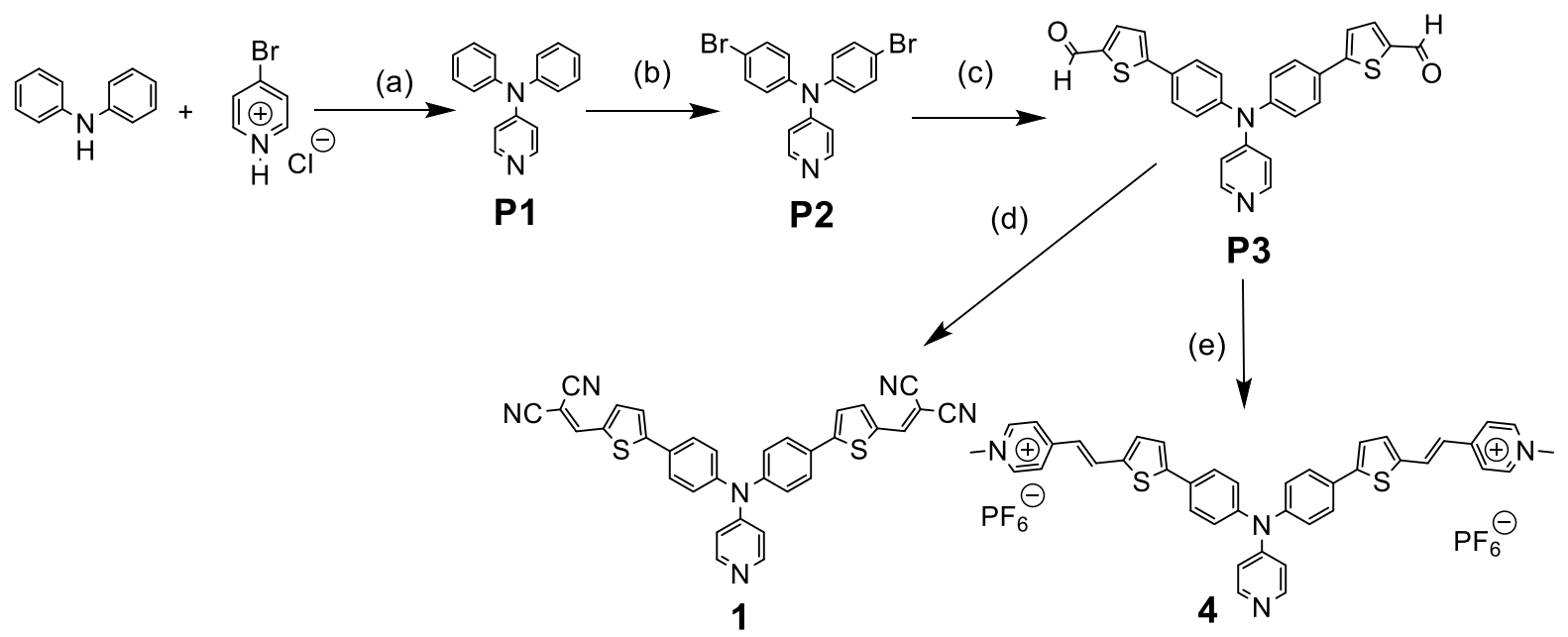

Scheme 1 Synthetic route to short pyridyl anchored dyes 1 and 4: (a) $\mathrm{Pd}(\mathrm{OAc})_{2},(t-\mathrm{Bu})_{3} \mathrm{P}, t-\mathrm{BuONa}$, toluene, $100{ }^{\circ} \mathrm{C}, 10 \mathrm{~h}, 34 \%$; (b) $\mathrm{NBS}, \mathrm{CHCl}_{3}$, reflux, overnight, 75\%; (c) 5-formyl-2-thiophene boronic acid, $\mathrm{Pd}\left(\mathrm{PPh}_{3}\right)_{4}, \mathrm{DME}, 2 \mathrm{M} \mathrm{Na}_{2} \mathrm{CO}_{3}$, reflux, 16h, 53\%; (d) malononitrile, pyridine, toluene, $70{ }^{\circ} \mathrm{C}, 16 \mathrm{~h}$, $60 \%$; (e) $\mathrm{N}$-methyl picolinium iodide, piperidine, $\mathrm{CH}_{3} \mathrm{Cl} / \mathrm{EtOH}, 80{ }^{\circ} \mathrm{C}, 4 \mathrm{~h}$, then $\mathrm{NH}_{4} \mathrm{PF}_{6}, 25 \%$. 


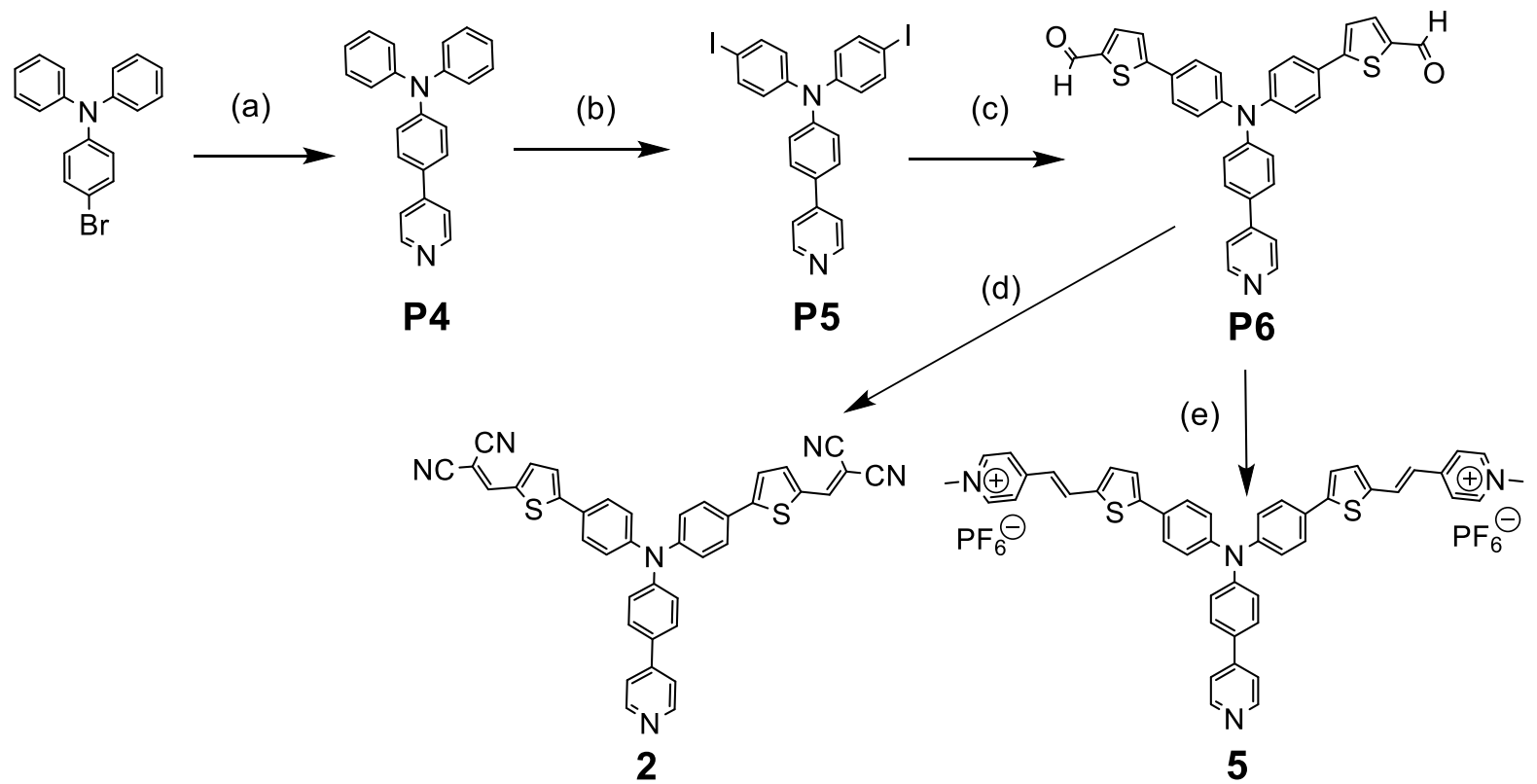

Scheme 2 Synthetic route to extended pyridyl anchored, bis acceptor dyes 2 and 5: (a) 4pyridinylboronic acid, $\mathrm{Pd}\left(\mathrm{PPh}_{3}\right)_{4}$, DME, $2 \mathrm{M} \mathrm{Na}_{2} \mathrm{CO}_{3}$, reflux, 16h, 77\% ; (b) $\mathrm{ICl}, \mathrm{Zn}(\mathrm{OAc})_{2}$, 1,4-dioxane, $\mathrm{RT}, 5 \mathrm{~h}, 91 \%$; (c) 5-formyl-2-thiophene boronic acid, $\mathrm{Pd}(\mathrm{dppf}) \mathrm{Cl}_{2}$, toluene/methanol, $\mathrm{K}_{2} \mathrm{CO}_{3}$, reflux, 16h, 56\%; (d) malononitrile, pyridine, toluene, $70{ }^{\circ} \mathrm{C}, 16 \mathrm{~h}, 75 \%$; (e) 4-methyl picolinium iodide, $\mathrm{CHCl}_{3} / \mathrm{EtOH}, 80{ }^{\circ} \mathrm{C}, 4 \mathrm{~h}, 45 \%$.
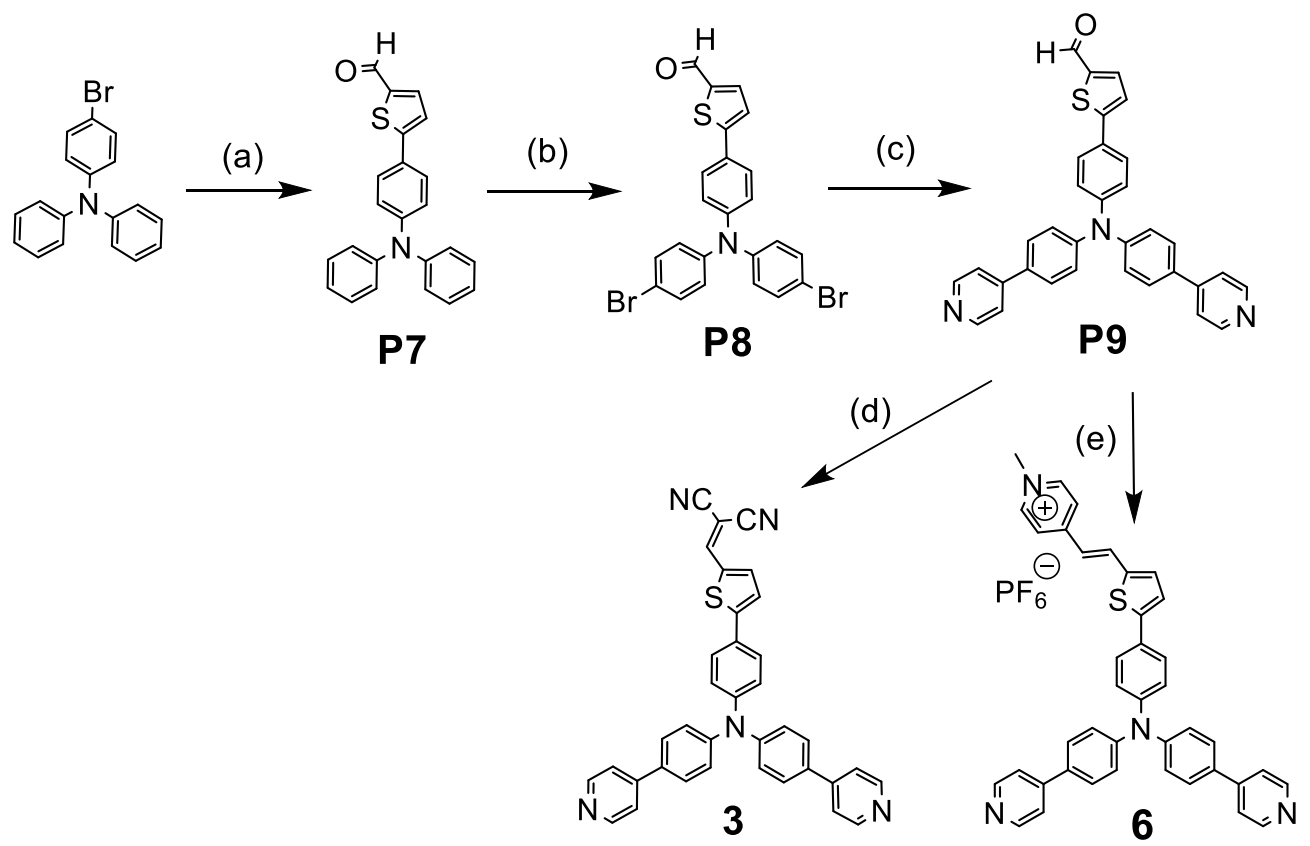

Scheme 3 Synthetic route to extended bis-pyridyl anchored, mono-acceptor dyes 3 and 6: (a) 5-formyl2-thiophene boronic acid, $\mathrm{Pd}(\mathrm{dppf}) \mathrm{Cl}_{2}$, toluene/methanol, $\mathrm{K}_{2} \mathrm{CO}_{3}$, reflux, $16 \mathrm{~h}, 89 \%$; (b) NBS, rt, $2 \mathrm{~h}$, 
95\%; (c) 4-pyridinylboronic acid, $\mathrm{Pd}\left(\mathrm{PPh}_{3}\right)_{4}, \mathrm{DME}, 2 \mathrm{M} \mathrm{Na}_{2} \mathrm{CO}_{3}$, reflux, $16 \mathrm{~h}, 65 \%$; (d) malononitrile, pyridine, toluene, $70{ }^{\circ} \mathrm{C}, 16 \mathrm{~h}, 75 \%$; (e) 4-methyl picolinium iodide, $\mathrm{CHCl}_{3} /$ ethanol, $80{ }^{\circ} \mathrm{C}, 4 \mathrm{~h}, 52 \%$.

The synthetic routes to $\mathbf{1}$ to $\mathbf{6}$ are shown in Schemes 1 to 3. Of the six dyes, five are new while $\mathbf{2}$ has been published before.[15] Our approach to $\mathbf{2}$ is different to the literature, however. Rather than making 4-diphenylamino-phenylboronic acid, we chose to shorten the synthesis by coupling commercially available 4-bromotriphenylamine and 4-pyridyl boronic acid via a standard Suzuki approach. The resulting pyridine derivative, P4, was then iodinated rather than brominated before reaction with formylthiophene boronic acid. From the resulting aldehyde, both dicyano (2) and pyridinium (5) acceptor dyes could be obtained by straightforward Knoevenagel condensations. Access to the other four dyes is also based on the same reaction of a formyl thiophene derivative (P3 or P9). In the case of the "short" dyes $\mathbf{1}$ and $\mathbf{4}$ (where the pyridine anchor is directly part of the triarylamine donor group) the starting point was $\mathrm{N}$-aryl coupling of diphenylamine with 4-bromopyridine hydrochloride to obtain P1. To access the bis-anchor, mono acceptor systems $\mathbf{3}$ and $\mathbf{6}$ the formylthiophene unit was introduced first, followed by the pyridines, rather than the other way round. This enabled the routes to $\mathbf{2}, \mathbf{3}, \mathbf{5}$, and $\mathbf{6}$ all to start from the same precursor, 4-bromotriphenylamine.

\section{Photophysical and Electrochemical Properties}

$U V$-vis absorption and emission: Absorption spectra of $\mathbf{1}$ to $\mathbf{6}$ in acetonitrile are shown in Fig. 2 (top). All six show two major absorption bands in the visible and near-UV. The stronger, lower energy band between 400 and $600 \mathrm{~nm}$ can be assigned to intramolecular charge trans fer (ICT) from the donor to the acceptor, while the higher energy band that has a peak at $c a .350$ $\mathrm{nm}$ can be assigned to $\pi-\pi^{*}$ electronic transitions. The $\lambda_{\max }$ of the ICT band shifts very significantly $(30-46 \mathrm{~nm})$ to the red upon replacing the pyridyl binding group of $\mathbf{1}$ and $\mathbf{4}$ with the extended phenyl pyridyl unit, likely because it reduces the electron withdrawing influence 
of the pyridyl ring on the donor group. Generally, the dicyano acceptor gives rise to a more red-shifted $\lambda_{\max }$ than the pyridinium acceptor (with the exception of the pyridyl systems $\mathbf{1}$ and 4), and this difference is significant in $\mathbf{2}$ vs $\mathbf{5}$, but the band has a broader shape in the pyridiniums and is also more intense ( $\varepsilon$ of up to $62300 \mathrm{M}^{-1} \mathrm{~cm}^{-1}, 12$ to $43 \%$ higher than for the comparable dicyano). Consequently, the pyridiniums absorb more strongly at long wavelengths, their absorption edge is at lower energy and lower $E_{0-0}$ values are found for these systems. Compared to other dyes with the indolium cationic acceptor,[16] however, $\lambda_{\max }$ for 5 is over $100 \mathrm{~nm}$ lower and the $\varepsilon$ lower by around 50\%, illustrating the effect of extended conjugation onto the larger indolium acceptor. The higher energy $\pi-\pi^{*}$ transition varies significantly in strength relative to the ICT band. In the pyridyl (rather than phenyl pyridyl) binder based systems $\mathbf{1}$ and $\mathbf{4}$, it is very weak, appearing only as a small bump, while in $\mathbf{3}$ and 6 (two phenyl pyridyl groups) its peak $\varepsilon$ exceeds that of the ICT band; the situation is intermediate (relative to ICT) for $\mathbf{2}$ and $\mathbf{5}$ which each have one phenyl pyridyl binding group. Thus, it appears this peak is associated with the pyridyl or phenyl pyridyl units. Emission spectra (Table 1, Figure S2) show red-shifts in $\lambda_{\mathrm{em}}$ from $\mathbf{1}$ to $\mathbf{2}$ and then $\mathbf{3}$ consistent with the shifts in the absorption maxima, the pyridinium dyes however are non-emissive.

Table 1. Optical and electrochemical parameters of $\mathbf{1}$ to $\mathbf{6}$.

\begin{tabular}{|c|c|c|c|c|c|c|c|}
\hline Dye & $\begin{array}{l}\lambda_{\max }^{a} \\
/ / \mathbf{n m}\end{array}$ & $\begin{array}{c}\varepsilon / \mathbf{1 0}^{3} \\
\mathbf{M}^{-1} \mathbf{c m}^{-1}\end{array}$ & $\begin{array}{c}\lambda_{\mathrm{em}}{ }^{a} / \\
\mathbf{n m}\end{array}$ & $\begin{array}{c}\mathbf{D}^{0} / \mathbf{D}^{+}, \boldsymbol{E}_{p a} \text { or } \\
\boldsymbol{E}_{1 / 2} \text { vs NHE / } \\
\mathbf{V}(\Delta \mathbf{E}, \mathbf{m V})\end{array}$ & $\begin{array}{c}\mathrm{D}^{0} / \mathrm{D}^{-}, E_{\mathrm{pc}} \text { or } \\
E_{1 / 2} v s \mathrm{NHE} / \\
\mathrm{V}(\Delta \mathrm{E}, \mathrm{mV})\end{array}$ & $\begin{array}{l}E_{0-0}^{b} \\
/ \mathrm{V}\end{array}$ & $\begin{array}{c}\text { Computed } \\
\text { HOMO-LUMO }^{c} \\
\text { eV }\end{array}$ \\
\hline 1 & 445 & 41.7 & 626 & 1.42 & -0.68 & 2.27 & 2.30 \\
\hline 2 & 491 & 46.8 & 673 & 1.32 & -0.72 & 2.07 & 2.12 \\
\hline 3 & 475 & 23.6 & 642 & $1.34(100)$ & -0.71 & 2.21 & 2.23 \\
\hline 4 & 449 & 45.3 & $-{ }^{d}$ & $-e^{e}$ & -0.63 & 2.19 & 2.11 \\
\hline 5 & 478 & 62.3 & $-{ }^{d}$ & 1.26 & $-0.52(120)^{f}$ & 1.99 & 1.90 \\
\hline 6 & 471 & 33.8 & $-{ }^{d}$ & $-{ }_{-e}^{e}$ & -0.69 & 2.10 & 2.04 \\
\hline
\end{tabular}

(a) Absorption and emission spectra recorded in $\mathrm{CH}_{3} \mathrm{CN}$ solution. (b) The HOMO-LUMO gap E E-0 estimated from the absorption edge. (c) Lowest energy TD-DFT calculated UV-vis transition (HOMO-LUMO), see experimental for details. (d) All three pyridinium dyes are non-emissive. (e) Dye oxidation peak cannot be clearly distingu ished from solvent oxidation. (f) Apparently quasi-reversible process overlapped with irreversible peak at $-0.68 \mathrm{~V}$. Electrochemical data were obtained using $0.1 \mathrm{M} \mathrm{NBu}_{4} \mathrm{BF}_{4}$ in DMF as electrolyte, with an analyte concentration of $c a .1 \mathrm{mM}$. Potentials referenced to internal $\mathrm{Fc} / \mathrm{Fc}^{+}$and converted to $\mathrm{NHE}$ using $\mathrm{Fc} / \mathrm{Fc}^{+}=0.69 \mathrm{~V}$ vs NHE . 

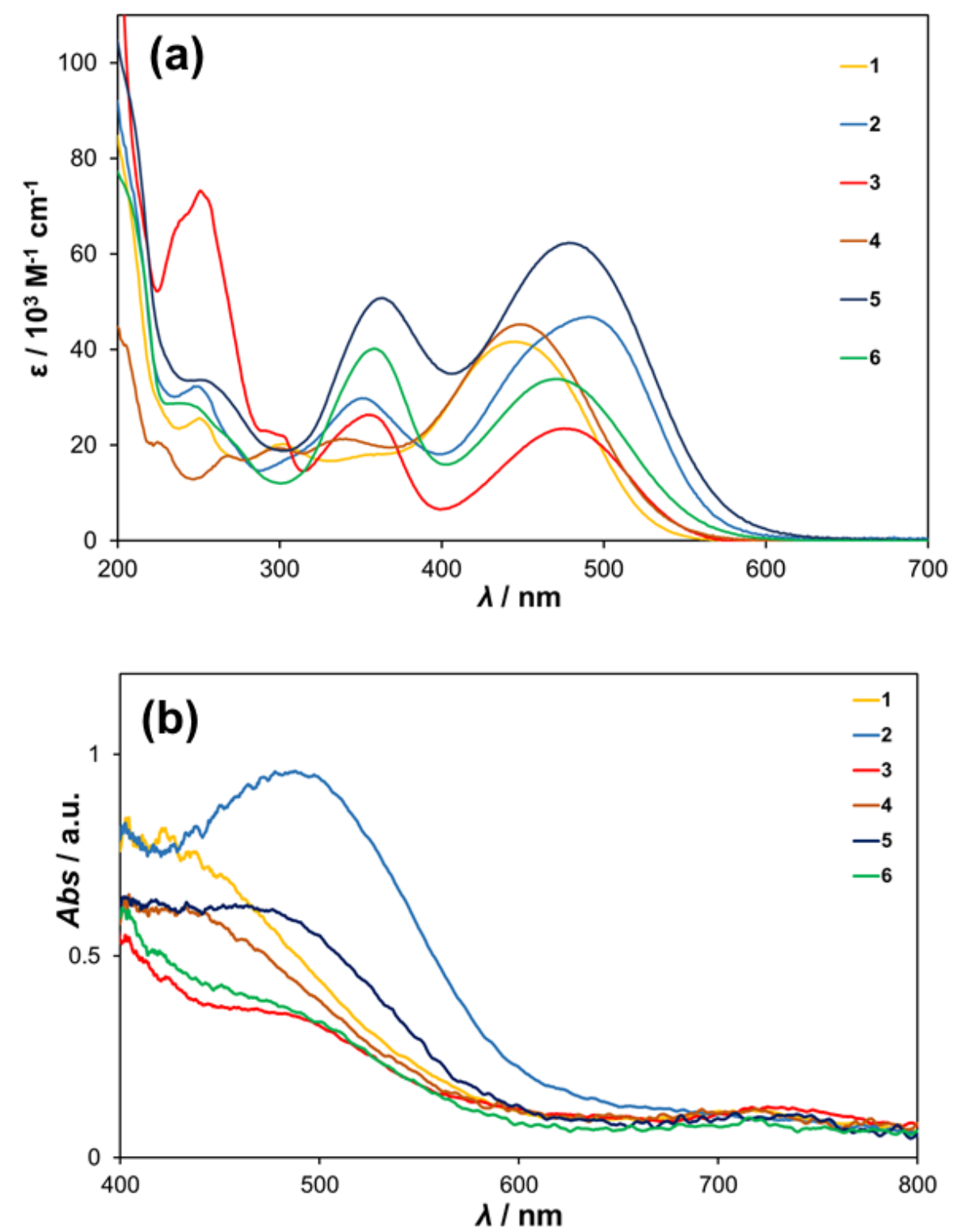

Fig. 2. Absorption spectra of $\mathbf{1}$ to 6. (a) In acetonitrile solutions at $298 \mathrm{~K}$. (b) Absorbed onto the NiO films used for solar cell studies.

Fig. 2 (bottom) shows the absorption spectra of the dyes on $\mathrm{NiO}$, by obtained by soaking $\mathrm{NiO}$ films in the dye solutions for 20 hours. The spectra are broader than in solution, and only $\mathbf{2}$ and 5 have clearly defined absorbance peaks on $\mathrm{NiO}$, at 487 and $463 \mathrm{~nm}$ respectively. These blue shifts could indicate electronic communication between the dyes and the $\mathrm{NiO}$ surface, although contribution from the $\mathrm{NiO}$ background is also possible. The absorbances obtained in the films are generally rather low and follow a different pattern to the solution extinction coefficients. 
With the short, pyridyl anchored $\mathbf{1}$ and $\mathbf{4}$, stronger light absorption is seen for dicyano-acceptor 1, despite a lower $\varepsilon$. Similarly, but more pronounced, the peak absorbance for dicyano $\mathbf{2}$ on $\mathrm{NiO}$ is over $50 \%$ greater than for pyridinium 5, even though its $\varepsilon$ is $25 \%$ lower. For the bisanchor dyes $\mathbf{3}$ and $\mathbf{6}$, there is little difference in light absorption. This implies that in every case significantly less of the cationic dye is adsorbed on the $\mathrm{NiO}$ surface, and the trend seems to be more dramatic for the dicationic species. This suggests it is likely a consequence of repulsion of the cationic dyes by the positive surface charge associated with $\mathrm{NiO}$, as well as the larger size of the pyridinium architectures.

Cyclic Voltammetry: Electrochemical data are also summarised in Table 1 and were obtained in $\mathrm{DMF}$, rather than $\mathrm{MeCN}$, as the compounds were not sufficiently soluble in $\mathrm{MeCN}$ at the concentrations required for electrochemistry. In most cases, oxidation of the donor and reduction of the acceptor appear irreversible on the timescale of these measurements, although quasi-reversiblity is seen in the oxidation of $\mathbf{3}$, and reduction of $\mathbf{5}$ (Fig. S1, SI). Moreover, for two of the three pyridinium compounds, the oxidation of the donor cannot be clearly observed above solvent background. This means that only a limited interpretation can be made of the data, but some trends are apparent. Firstly, in all cases the reduction potential of the pyridinium dye is less negative than its nitrile counterpart, implying that it is a stronger acceptor and electron transfer to it is easier. This is consistent with the smaller HOMO-LUMO (E00) gaps estimated for the pyridiniums from the absorption edges of $\mathbf{4}$ to $\mathbf{6}$, though not the blue shifted $\lambda_{\max }$ values. Interestingly, though, for the only pyridinium with a clearly observed oxidation potential (5), the oxidation potential is less positive than for any of the nitrile systems. This is consistent with the low $E_{00}$ observed for this compound. The nature of the binder also seems to influence the potential of the HOMO (donor), with the highest oxidation potential being seen for 1, where the electron deficient ring is directly connected to the aryl amine N. In all cases, 
the dye oxidation potentials are comfortably more positive than the top of the NiO VB $(0.54 \mathrm{~V}$ vs $\mathrm{NHE}$ ), indicating that hole transfer to $\mathrm{NiO}$ is energetically favourable - similarly, the reduction potentials of the dye acceptors are comfortably more negative than that of the $\mathrm{I}^{-} / \mathrm{I}_{3^{-}}$ redox electrolyte.

DFT calculations: DFT and TD-DFT calculations were performed on $\mathbf{1}$ to $\mathbf{6}$ using the Amsterdam Density Functional (ADF) software package, with the B3LYP functional and DZP basis set.[22-24]. Acetonitrile solvent was added using COSMO. The trend in the lowest energy TD-DFT calculated transitions (all HOMO $\rightarrow$ LUMO) perfectly matches that of the experimental $E_{00}$ values within the individual dicyano ( $\mathbf{1}$ to $\mathbf{3}$ ) and pyridinium (4 to 6 ) series (Table 1), and also mirrors experimental findings by showing that the pyridinium HOMO $\rightarrow$ LUMO transitions are in all cases lower energy than those of their dicyano analogues. However, the computed lowering in transition energy for pyridiniums $(0.19$ to $0.22 \mathrm{eV})$ is greater than that estimated from the UV-vis and electrochemical measurements (0.08 to 0.11 $\mathrm{eV}$ ) - this is reflected in the in the position of the computed lowest energy transitions which are always a few nanometres to the blue of the measured dye absorption edge for the dicyano dyes (Fig. S3, SI). The computed spectra also echo experiment by consistently finding higher oscillator strengths $\left(f_{\text {os }}\right)$ for the pyridinium dyes, which are shown to have higher extinction coefficients.

Analysis of the orbital-to-orbital transitions (Tables S1 to S6, Figures S4 to S9, SI) indicates that the two families of dyes have fundamentally similar electronic structures. In all cases, the lowest energy (and usually strongest) transition involves charge transfer (CT) from a HOMO based on the triarylamine unit, to a LUMO largely based on the thiophene bridge and dicyano or pyridinium acceptor. The highest energy transitions are largely $\pi \rightarrow \pi^{*}$ in character. However, 
the structural variations lead to differences in the spatial distribution and energies of the computed orbitals which are consistent with the observed spectroscopic properties (vide supra) and solar cell performance (vide infra) of the dyes.

Firstly, in both the dicyano and pyridinium series, clear trends can be seen on moving from the mono-pyridine $(\mathbf{1}, \mathbf{4})$, to mono-phenylpyridine $(\mathbf{2}, \mathbf{5})$ and then bis-phenylpyridine binding units. In 1 and 4 (Tables S1, S4; Fig.s S4, S7), the HOMO levels extend onto the electron deficie nt pyridine ring, lowering their energy and resulting in larger HOMO-LUMO gaps (by at least $0.07 \mathrm{eV}$, Table 1) than the other dyes in each series. In 2 and 5 (Tables S2, S5; Fig.s S5, S8), however, the phenyl spacer reduces the influence of the pyridine group on the triarylamine donor and participation of the pyridine ring is absent not only from the HOMO, but also the HOMO-1 and HOMO-2. Adding a second phenylpyridine binding unit can be expected to have a detrimental effect on charge separation performance (as found experimentally) because in both 3 and 6 (Tables S3, S6; Fig.s S6, S9) their presence introduces new acceptor orbitals at accessible energies (LUMO+1/+2 for $3, \mathrm{LUMO}+2$ for $\mathbf{6})$ that are expected to interact strongly with the NiO surface. The excited state HOMOs (Fig.s S6, S9) are also located on the phenylpyridine binding groups. This will encourage recombination, moreover in both dyes there are calculated CT transitions in the near UV from the HOMO, to these orbitals -sending electrons in an undesired direction. This is consistent with the experimentally observed, comparatively stronger absorption between 300 and $400 \mathrm{~nm}$ for 3 and $\mathbf{6}$. 

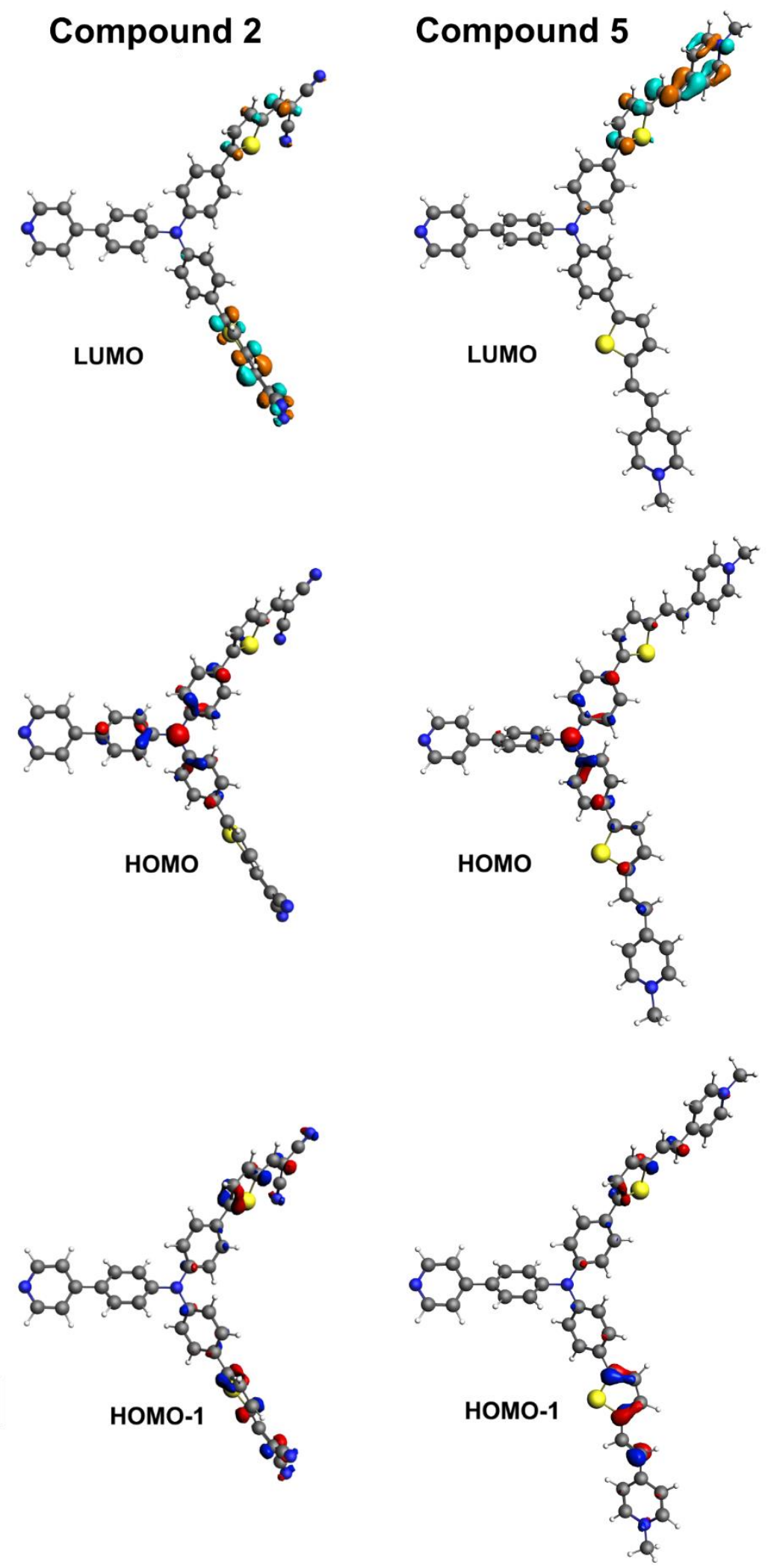

Fig. 4. Selected DFT calculated frontier orbitals of $\mathbf{2}$ and $\mathbf{5}$ 
Secondly, analysis of the orbitals involved in the computed UV-vis transitions reveals likely advantages for the pyridinium acceptor. For both acceptor systems, the LUMOs generally involve a combination of the acceptor, and thiophene bridge (Fig. 4). For the pyridiniums, this LUMO is lower energy (leading to transitions covering more of the visible spectrum), and larger due to the increased size of the pyridinium, vs dicyano group. Thus, the pyridinium is able to delocalise charge further away from the triarylamine donor and $\mathrm{NiO}$ surface. The pyridiniums also show less participation of the acceptor in the HOMO and other donor orbitals (HOMO-1/-2), thus, the pyridinium systems are likely to separate charge more effectively and direct it away from the surface of the p-type substrate. Participation of the relatively electron deficient pyridine/phenylpyridine units in the HOMO orbitals is also suppressed in the pyridiniums, raising the HOMO energy and helping absorb more visible light. Lastly, in $\mathbf{5}$ and 6 (vs 2 and 3), there is less tendency to transfer charge to the phenylpyridines - in compound 5 the $\mathrm{LUMO} /+1 /+2$ are all based on the pyridinium and only the LUMO+3 involves the phenylpyridine (Fig. S8) whereas in 2 the LUMO+2 is phenylpyridine based (Fig. S5), and the transition to it is about three times stronger than the equivalent in 2 . A similar situation pertains for 6 vs 3 (See SI, Tables S3 and S6, Fig.s S6 and S9).

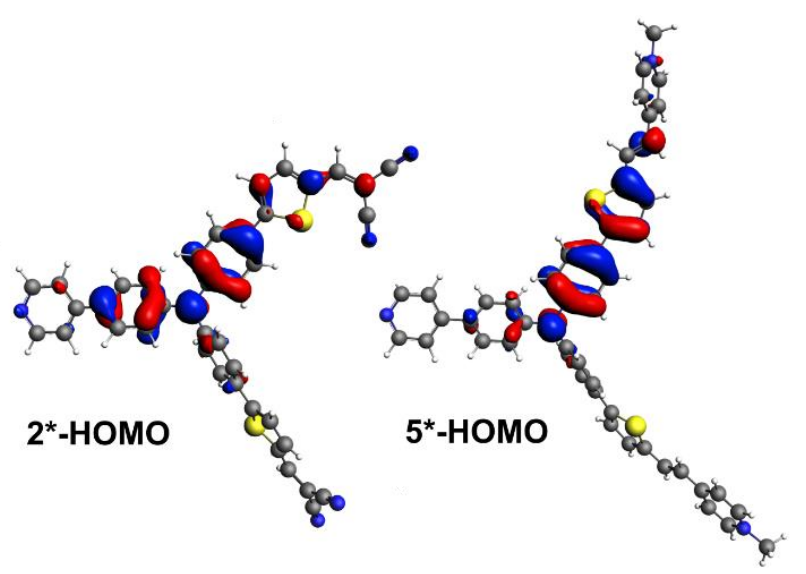

Fig. 5. DFT calculated HOMOs of the excited states of $\mathbf{2}$ and $\mathbf{5}$. It can be seen that in $\mathbf{5}$, less electron density remains on the phenylpyridine unit, and more on the bridge and the acceptor, providing more effective charge separation. 
Calculation of the structures of the first excited states reflects these findings, through excited state HOMOs for the pyridiniums that tend to distribute less electron density onto the pyridine/phenylpyridine binding groups, and more onto the bridge and acceptor (Fig.s S4 to S9). This is seen most clearly for the excited states of 5 vs 2 (Fig. 5). Computed ground-toexcited state dipole moment changes (Table S7) shows that in every pyridinium/dicyano pair, charge is moved further away from the donor (and thus the NiO surface) by the pyridinium. Thus, electronically the pyridinium group appears to have the advantage over the dicyano for light absorption and charge separation.

\section{Photovoltaic performance}

Table 2. Photovoltaic parameters of NiO p-DSSCs based on 1 to $\mathbf{6}$.

\begin{tabular}{|c|c|c|c|c|c|}
\hline Dye & Loading / 10 ${ }^{-10} \mathrm{~mol}^{a}$ & $J_{\mathrm{sc}} / \mathrm{mA} \mathrm{cm}^{-2}$ & $V_{\mathrm{oc}} / \mathrm{mV}$ & FF (\%) & $\eta(\%)$ \\
\hline 1 & 6.38 & 0.83 & 50 & 43 & 0.018 \\
\hline 2 & 11.8 & 1.6 & 103 & 36 & 0.060 \\
\hline 3 & 5.14 & 0.87 & 49 & 32 & 0.014 \\
\hline 4 & 3.30 & 0.83 & 66 & 33 & 0.018 \\
\hline 5 & 6.23 & 1.11 & 86 & 37 & 0.036 \\
\hline 6 & 4.96 & 0.84 & 70 & 23 & 0.014 \\
\hline
\end{tabular}

Electrolyte: $0.1 \mathrm{M} \mathrm{I}_{2}, 1 \mathrm{M} \mathrm{LiI}$ in MeCN. Counter electrode: platinized FTO glass. ${ }^{a}$ Loading per $0.25 \mathrm{~cm}^{2}, 1 \mu \mathrm{M}$ thick film, average of four films.

Photovoltaic parameters of devices based on the six sensitizers on $\mathrm{NiO}$ (with $\mathrm{I}^{-} / \mathrm{I}_{3}{ }^{-}$), under $\mathrm{AM}$ 1.5 solar simulated light, are shown in Table 2 (for $J$ - $V$ curves see Fig. S10, SI) and IPCE spectra are displayed in Fig. 6. In our work, compound $\mathbf{2}$ shows a slightly weaker performance $(\eta=0.06 \%)$ than previously published in similar conditions $(\eta=0.093 \%),[15]$ due to a lower $J_{\mathrm{sc}}$, but is still the best performing dye in this series. The next best performance $(\eta=0.036 \%)$ comes from the other phenyl pyridyl, bis-acceptor structure $\mathbf{5}$, which has both lower $J_{\mathrm{sc}}$ and lower Voc than 2, and weaker IPCE across the spectrum from 400 to $700 \mathrm{~nm}$. The obvious 
explanation is a lower loading of $\mathbf{5}$, indicated by the weaker absorption of the $\mathrm{NiO}$ films prepared with this compound (Fig. 2) and confirmed in a loading study (Table 2, Fig. S11). Otherwise, the higher extinction coefficient of $\mathbf{5}$ coupled with its similar redox properties (and the better electronic structure for charge separation suggested by DFT, vide supra) might be expected to yield a higher power conversion efficiency. Indeed, absorbed photon to current efficiencies (APCEs, Fig. S12, SI) shows a very similar performance for $\mathbf{5}$ vs $\mathbf{2}$ across the visible range. This suggests that the performance of $\mathbf{5}$ in p-DSSCs is held back by the ability to load dicationic dyes onto the positively charged $\mathrm{NiO}$ surface, rather than the fundamental electronics and light absorption properties of the dye.

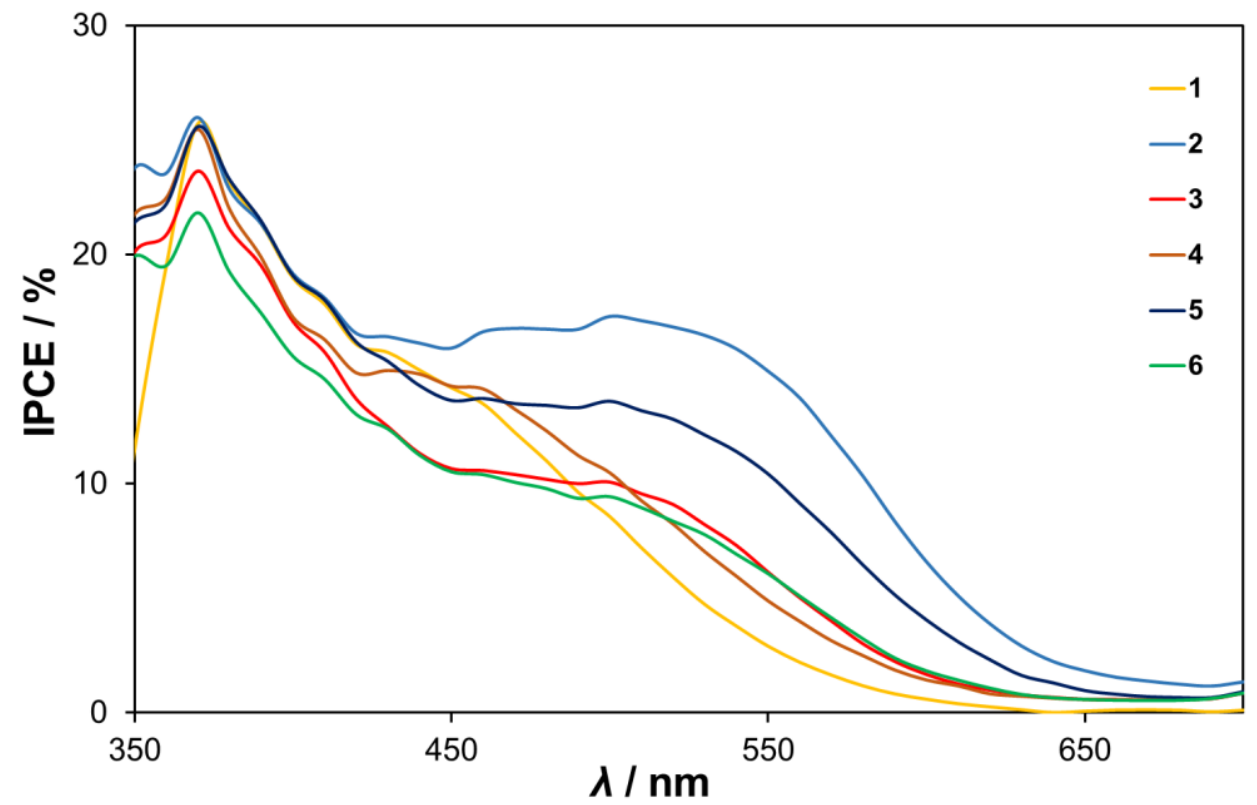

Fig. 6. IPCE spectra of p-DSSCs assembled with 1 to $\mathbf{6}$

The other dyes $(\mathbf{1}, \mathbf{3}, \mathbf{4}$ and $\mathbf{6})$ produce overall efficiencies at best $50 \%$ of that of $\mathbf{5}$. This is likely because addition of a second anchoring group (as in $\mathbf{3}$ and $\mathbf{6}$ ) in place of an acceptor sacrifices light absorption, as seen in the UV-vis spectra in solution and on the $\mathrm{NiO}$ surface and reflected in the IPCE spectra. In addition, the DFT calculations suggest that the additional phenylpyridine leads to these anchoring groups acting as acceptors, close to and interacting 
strongly with the NiO surface. The same absorption profile argument, and weakened donor capability in the triphenylamine, can be used to explain the weak performance of $\mathbf{1}$ and $\mathbf{4}$-note that $\mathbf{4}$ also has the lowest loading of any of the dyes. In addition, these systems all show lower VOC than seen for $\mathbf{2}$ or $\mathbf{5}$, which could imply that these dyes, that are either shorter or have fewer/less optimally positioned electron acceptor groups, do less to mitigate recombination than $\mathbf{2}$ or $\mathbf{5}$ - again consistent with the DFT calculated electronic structures. It is also consistent with the prior observation that extension of $\mathbf{2}$ with a further phenyl spacer increases $V_{\mathrm{OC}}$ by $c a$. $20 \mathrm{mV}$.[15] Lastly the pyridinium systems $\mathbf{4}$ and $\mathbf{6}$, despite the clearly lower dye loading of $\mathbf{4}$, yield higher $V_{\mathrm{OCS}}$ (and very similar $J_{\mathrm{SCS}}$ ) to their dicyano counterparts $\mathbf{1}$ and $\mathbf{3}$, although $\eta$ values are identical due to lower fill factors. IPCEs for $\mathbf{4}$ are slightly higher than for $\mathbf{1}$, and for $\mathbf{6}$ and $\mathbf{3}$ are very similar. Conversion to APCE (Fig. S12) shows a clear advantage for $\mathbf{4}$ over $\mathbf{1}$ across the visible range, and for $\mathbf{6}$ over $\mathbf{3}$ at longer wavelengths. Thus, the overall picture from the solar cell measurements suggests that the simple pyridinium acceptor performs just as well, or better than the widely used dicyano group in p-DSSCs, but that in the specific example here, inadequate dye loading (around 50\% lower for bis-acceptor, dicationic systems vs dicyano analogues) holds back performance. As the difference in loading is much smaller for the monoacceptor, mono-cationic $\mathbf{6}$ compared to its dicyano analogue, loadings would likely improve in systems with carboxylate binders where deprotonation can mitigate repulsion between positively charged dyes, and the positively charged $\mathrm{NiO}$ surface.

\section{Conclusion}

In conclusion, five new sensitizer dyes with pyridyl binding groups have been synthesised, and a shortened route developed to a pre-existing one. In these systems, light absorption is enhanced by use of pyridinium, rather than dicyano acceptor groups, as seen through broader spectrum light absorption and higher extinction coefficients. In addition, DFT and TD-DFT 
calculations suggest that the electronic structure of the pyridinium dyes is better set up to transfer charge away from the substrate in a p-DSSC. The device performance of the pyridinium dyes however does not exceed that of their dicyano counterparts, and appears to be held back by weaker dye absorption on the substrate - potentially a consequence of the positively charged acceptor and positively charged substrate, and thus potentially mitigated by use of binders that adopt a negative charge (such as carboxylate). Therefore, with differe nt binders pyridiniums could provide a new, easily accessible and versatile class of acceptor for use in p-DSSCs. The results of this study also show that using two pyridine anchors rather than one, or attaching the pyridine anchor directly to the $\mathrm{N}$-donor, degrades performance in the $\mathrm{p}$ DSSC due to poorer light absorption and the ability of the pyridine to act as an acceptor that interacts strongly with the p-type substrate.

\section{Acknowledgements}

We thank the UK EPSRC National Mass Spectrometry Facility for MS. ARM thanks the EU for Marie Skłodowska Curie Fellowship No. 656658 'NanoCuI", and JF the Royal Society for Research Grant RG140578. EAG and FAB acknowledge the EPSRC and Newcastle University for funding studentship EPJ5002881, EAG and JM thank the EU for ERC starting grant pTYPE 715354.

\section{Data Sharing Statement}

In addition to the supplementary information data can be accessed by contacting the corresponding author. 


\section{References}

[1] O’Regan B, Grätzel M. A low-cost, high-efficiency solar cell based on dye-sensitized colloidal TiO2 films. Nature 1991;353:737-40.

[2] Grätzel M. Perspectives for dye-sensitized nanocrystalline solar cells. Prog Photovoltaics $2000 ; 8: 171-85$.

[3] Grätzel, M. Photoelectrochemical cells. Nature 2001;414:338-44

[4] Mathew S, Yella A, Gao P, Humphry-Baker R, Curchod BF, Ashari-Astani N, Tavernelli I, Rothlisberger U, Nazeeruddin MK, Grätzel M. Dye-sensitized solar cells with 13\% efficiency achieved through the molecular engineering of porphyrin sensitizers. Nat Chem $2014 ; 6: 242-7$.

[5] Hagfeldt A, Boschloo G, Sun L, Kloo L, Pettersson H. Dye sensitized solar cells. Chem Rev 2010;110:6595-663.

[6] Odobel F, Le Pleux L, Pellegrin, Y, Blart E. New photovoltaic devices based on the sensitization of p-type semiconductors: challenges and opportunities. Acc Chem Res 2010;43:1063-71

[7] Odobel F, Pellegrin Y, Gibson EA, Hagfeldt A, Smeigh AL, Hammarström L. Recent advances and future directions to optimize the performances of p-type dye-sensitized solar cells. Coord Chem Rev 2012;256:2414-23.

[8] Odobel F, Pellegrin Y. Recent advances in the sensitization of wide-band gap nanostructured p-type semiconductors. Photovoltaic and photocatalytic applications. J Phys Chem Lett 2013;4:2551-64. 
[9] Gibson EA, Smeigh AL, Le Pleux L, Hammarström L, Odobel F, Boschloo G, Hagfeldt A. Cobalt polypyridyl-based electrolytes for p-type dye-sensitized solar cells J Phys Chem C 2011;115:9772-79.

[10] Powar S, Daeneke T, Ma MT, Fu D, Duffy NW, Götz G, Weidelener M, Mishra A, Bäurle P, Spiccia L, Bach U. Highly efficient p-type dye-sensitized solar cells based on tris $(1,2-$ diaminoethane)cobalt(II)/(III) electrolytes. Angew Chem Int Ed 2013;52:602-5.

[11] Perera IR, Daeneke T, Makuta S, Yu Z, Tachibana Y, Mishra A, Bäuerle P, Ohlin AC, Bach U, Spiccia L. Application of the tris(acetylacetonato)iron(III)/(II) redox couple in p-type dye sensitized solar cells. Angew Chem Int Ed 2015;54:3758-62.

[12] Gibson EA, Smeigh AL, Le Pleux L, Fortage J, Boschloo G, Blart E, Pellegrin Y, Odobel F, Hagfeldt A, Hammarström L. A p-type NiO-based dye-sensitized solar cell with an opencircuit voltage of $0.35 \mathrm{~V}$. Angew Chem Int Ed 2009;48:4402-5.

[13] Zhang XL, Zhang Z, Chen D, Bauerle P, Bach U, Cheng YB. Sensitization of nickel oxide: improved carrier lifetime and charge collection by tuning nanoscale crystallinity. Chem Commun 2012;48:9885-7.

[14] See for example: (a) Nattestad A, Mozer J, Fischer MKR, Cheng Y-B, Mishra A, Bäuerle P, Bach U. Highly efficient photocathodes for dye-sensitized tandem solar cells. Nature Mater 2010;9:31-35. (b) Farré Y, Raissi M, Fihey A, Pellegrin Y, Blart E, Jacquemin D, Odobel F. A blue diketopyrrole sensitizer with high efficiency in nickel-oxide-based dye-sensitized solar cells. ChemSusChem 2017;10:2618-2625.

[15] Cui J, Lu J, Xu X, Cao K, Wang Z, Alemu G, Yuang H, Shen Y, Xu J, Cheng Y, Wang M. Organic sensitizers with pyridine ring anchoring group for $\mathrm{p}$-type dye-sensitized solar cells. J Phys Chem C 2014;118:16433-40. 
[16] Wood CJ, Summers GH, Gibson EA. Increased photocurrent in a tandem dye-sensitized solar cell by modifications in push-pull dye design. Chem Commun 2015;51:3915-18.

[17] Coe BJ, Harris JA, Asselberghs I, Clays K, Olbrechts G, Persoons A, Hupp JT, Johnson RC, Coles SJ, Hursthouse SJ, Nakatani K. Quadratic nonlinear optical properties of N-aryl stilbazolium dyes. Adv Funct Mater 2002;12:110-7.

[18] Zhao CF, He GS, Bhawalkar JD, Park CK, Prasad PN. Newly synthesized dyes and their polymer/glass composites for one and two-photon pumped solid-state cavity lasing. Chem Mater 1995;7:1979-83.

[19] Li Y, Ren T, Dong WJ. Tuning photophysical properties of triphenylamine and aromatic cyano conjugate-based wavelength-shifting compounds by manipulating intramolecular charge transfer strength. J Photochem Photobiol A 2013;251:1-9.

[20] Hagberg DP, Jiang X, Gabrielsson E, Linder M, Marinado T, Brinck T, Hagfeldt A, Sun L. Symmetric and unsymmetric donor functionalization. Comparing structural and spectral benefits of chromophores for dye-sensitized solar cells. J Mater Chem 2009;19:7232-38.

[21] Ibrahim SK, PhD Thesis, University of Sussex 1992.

[22] (a) Te Velde G, Bickelhaupt FM, Baerends EJ, Fonseca Guerra C, van Gisbergen SJA, Snijders JG, Ziegler T. Chemistry with ADF. J Comput Chem 2001;22:931-967. (b) Fonseca Guerra C, Snijders JF, Te Velde G, Baerends E. Towards an order-N DFT method. J Theor Chem Acc 1998;99:391-403. (c) ADF2017.110, SCM, Theoretical Chemistry, Vrije Universiteit, Amsterdam, The Netherlands, http://www.scm.com.

[23] Stephens PJ, Devlin FJ, Chabalowski CF, Frisch MJ. Ab initio calculation of vibrational absorption and circular dichroism spectra using density functional force fields. J Phys Chem 1994;98:11623-11627. 
[24] van Gisbergen SJA, Snijders JG, Baerends EJ. Implementation of time-dependent denisty functional response equations. Comput. Phys. Commun. 1999;118:119-138. 